Some Improved Error Estimates for the Modified Method of Characteristics

by

C.N. Dawson

T.F. Russell

M.F. Wheeler

Technical Report 88-2, January 1988

(Revised September 12, 1988) 



\title{
Some Improved Error Estimates for the Modified Method of Characteristics
}

\author{
C. N. Dawson ${ }^{1}$, T. F. Russell ${ }^{2}$, and M. F. Wheeler ${ }^{1}$
}

Dedication: To Professor Jim Douglas, Jr. on the occasion of his 60th birthday.

\section{Introduction}

The modified method of characteristics (MMOC) was first formulated for a (possibly nonlinear multidimensional) scalar parabolic equation by J. Douglas and T. F. Russell in [3] and then extended by Russell [10] to nonlinear coupled systems in two and three spatial dimensions. Similar schemes have been defined by Pironneau [9] for the incompressible Navier-Stokes equations and by Süli [12] and Morton, Priestley, and Süli [7] for first order hyperbolic equations, with the latter technique being referred to as the Euler characteristic Galerkin method. The intent of the method is to obtain accurate approximations to advection-dominated problems.

Basically, in the modified method of characteristics, one combines the time derivative and the advection term as a directional derivative. In other words, the procedure involves time-stepping along the characteristics, allowing one to use large accurate time-steps. An algorithm combining the mixed finite element method and the modified method of characteristics was first applied to the miscible displacement problem in porous media by Ewing, Russell, and Wheeler [5]. Recently, the scheme has been extended by Wheeler and Dawson [14] to advection-diffusion-reaction problems. Numerical results have verified that large accurate time steps are possible, and sharp fronts have been resolved (without oscillations or numerical diffusion) by coarser grids than standard procedures can use.

In this paper, we consider the numerical solution of advection-diffusion problems of the following form:

$$
\left.\begin{array}{ll}
-\nabla \cdot\left(\frac{k(\mathbf{x})}{\mu(c)} \nabla p\right) \equiv \nabla \cdot \mathbf{u}=q(\mathbf{x}, t), & \mathbf{x} \in \Omega, t \in J, \\
\phi \frac{\partial c}{\partial t}-\nabla \cdot D(\mathbf{u}) \nabla c+\mathbf{u} \cdot \nabla c=\tilde{q}(\tilde{c}-c), & \mathbf{x} \in \Omega, t \in J, \\
c(\mathbf{x}, 0)=c_{0}(\mathbf{x}), & \mathbf{x} \in \Omega, \\
\mathbf{u} \cdot \eta=D(\mathbf{u}) \nabla c \cdot \eta=0, & \mathbf{x} \in \partial \Omega,
\end{array}\right\}
$$

\footnotetext{
${ }^{1}$ Dept. of Mathematical Sciences, Rice University

${ }^{2}$ Dept. of Mathematics, University of Colorado at Denver
} 
where $\Omega$ is a bounded domain in $\Re^{2}, J=(0, T]$, and $\tilde{q}=\max (q, 0)$ is nonzero at source points only. Here $\eta$ is the outward normal to $\Omega . D(\mathbf{u})$ is a tensor matrix and can be velocity-dependent. For $\mathbf{u}=\left(u_{x}, u_{y}\right), D$ generally has the form

$$
D(\mathbf{u})=D_{m} I+\frac{\alpha_{l}}{|\mathbf{u}|}\left[\begin{array}{cc}
u_{x}^{2} & u_{x} u_{y} \\
u_{x} u_{y} & u_{y}^{2}
\end{array}\right]+\frac{\alpha_{t}}{|\mathbf{u}|}\left[\begin{array}{cc}
u_{y}^{2} & -u_{x} u_{y} \\
-u_{x} u_{y} & u_{x}^{2}
\end{array}\right],
$$

where $D_{m}$ is the molecular diffusivity and $\alpha_{l}$ and $\alpha_{t}$ are the longitudinal and transverse dispersivities, respectively. Furthermore, in (1.1) $\tilde{c}$ is specified at injection wells and $\tilde{c}=c$ at production wells. Problems of this form arise in the study of reservoir simulation and contaminant flows [5, 14].

For convenience, we assume (1.1) is $\Omega$-periodic; i.e., we assume all functions in (1.1) are spatially $\Omega$-periodic. This is physically reasonable, since no-flow boundaries are generally treated by reflection, and because in general interior flow patterns are much more important than boundary effects. Thus, the no-flow boundary conditions above can be dropped.

This paper is divided into three additional sections. In the second section we establish notation. The modified method of characteristics for linear advection problems with $\mathbf{u}$ assumed to be given, is treated in Section 3. The two major results of this section involve derivation of a suboptimal convergence result for the case $D(\mathbf{u}) \geq 0$ (diffusion-dispersion can be zero) and an $L^{2}\left(L^{2}\right)$ error estimate which involves optimal rate and improved (virtually optimal) norms on the solution. The results of Section 3 are generalized in Section 4 to include combining a mixed finite element approximation to $(p, \mathbf{u})$ with the MMOC for approximating $c$. Here a suboptimal estimate is obtained with restrictions on the order of the approximating spaces. Improved norm estimates in $L^{2}\left(L^{2}\right)$ are also derived for $\mu$ independent of $c$.

We wish to remark that by 'virtually optimal' we mean that the norm estimates are of the form

$$
\sum_{n=1}^{N^{*}}\|c-C\|^{2} \Delta t \leq K h^{2(r+1)}\left(\sum_{n=1}^{N^{*}}\|c\|_{r+1}^{2} \Delta t+\left\|c_{t}\right\|_{L^{2}\left(H^{r-1}\right)}^{2}+\mathcal{O}\left(\Delta t^{2}\right)\right) .
$$

Here the exponent on $h$ cannot be improved.

\section{Preliminaries and notation}

On $\Omega$, we define the following Sobolev spaces and norms:

$$
\begin{array}{cc}
L^{2}(\Omega)=\left\{f: \int_{\Omega}|f|^{2} d \mathbf{x}<\infty\right\}, & \|f\|^{2}=\int_{\Omega}|f|^{2} d \mathbf{x}, \\
L^{\infty}(\Omega)=\left\{f: \sup _{\Omega}|f|<\infty\right\}, & \|f\|_{\infty}=\sup _{\Omega}|f|,
\end{array}
$$




$$
\begin{array}{r}
H^{m}(\Omega)=\left\{f: \frac{\partial^{|\alpha|} f}{\partial \mathbf{x}^{\alpha}} \in L^{2}(\Omega) \text { for }|\alpha| \leq m\right\}, \\
\|f\|_{m}^{2}=\sum_{|\alpha| \leq m}\left\|\frac{\partial^{|\alpha|} f}{\partial \mathbf{x}^{\alpha}}\right\|^{2}, \quad m \geq 0, \\
W_{\infty}^{m}(\Omega)=\left\{f: \frac{\partial^{|\alpha|} f}{\partial \mathbf{x}^{\alpha}} \in L^{\infty}(\Omega) \text { for }|\alpha| \leq m\right\}, \\
\|f\|_{W_{\infty}^{m}}=\max _{|\alpha| \leq m}\left\|\frac{\partial^{|\alpha|} f}{\partial \mathbf{x}^{\alpha}}\right\|_{\infty}, \quad m \geq 0 .
\end{array}
$$

Also, we define

$$
H(\operatorname{div} ; \Omega)=\left\{\mathbf{f}=\left(f_{x}, f_{y}\right): f_{x}, f_{y}, \nabla \cdot \mathbf{f} \in L^{2}(\Omega)\right\},
$$

with

$$
\|\mathbf{f}\|_{H(d i v)}^{2}=\left\|f_{x}\right\|^{2}+\left\|f_{y}\right\|^{2}+\|\nabla \cdot \mathbf{f}\|^{2} .
$$

When $L^{2}$ is replaced by $H^{m}$ in the definition of $H(d i v)$, denote the resulting space by $H^{m}($ div $)$. The inner product on $L^{2}(\Omega)$ is denoted by $(\cdot, \cdot)$. Also note that $W_{\infty}^{0}=L^{\infty}$ and $H^{0}=L^{2}$.

We also use the following spaces that incorporate time dependence. Let $[a, b] \subset J$ and let $X$ be any of the above $L^{p}$ or Sobolev spaces. For $f(\mathbf{x}, t)$ suitably smooth on $\Omega \times[a, b]$, we let

$$
\begin{array}{r}
H^{m}(a, b ; X)=\left\{f: \int_{a}^{b}\left\|\frac{\partial^{\alpha} f(\cdot, t)}{\partial t^{\alpha}}\right\|_{X}^{2} d t<\infty, \quad \alpha \leq m\right\}, \\
\|f\|_{H^{m}(a, b ; X)}=\left(\sum_{\alpha=0}^{m} \int_{a}^{b}\left\|\frac{\partial^{\alpha} f(\cdot, t)}{\partial t^{\alpha}}\right\|_{X}^{2} d t\right)^{\frac{1}{2}}, \quad m \geq 0 .
\end{array}
$$

Similarly, $W_{\infty}^{m}(a, b ; X)$ and the norm $\|f\|_{W_{\infty}^{m}(a, b ; X)}$ are defined. If $[a, b]=\bar{J}$, we simplify our notation and write $L^{\infty}\left(W_{\infty}^{1}\right)$ for $L^{\infty}\left(0, T ; W_{\infty}^{1}(\Omega)\right)$, etc.

Let $\Delta t>0$. Set $t^{n}=n \Delta t$, and let $t^{N^{*}}=T$. Let $f^{n}=f\left(t^{n}\right)$. We define, for $1 \leq p<\infty$, the discrete time-dependent norms

$$
\|\| f\left\|_{L^{p}\left(H^{k}\right)}^{p} \equiv \sum_{n=1}^{N^{*}}\right\| f^{n} \|_{k}^{p} \Delta t
$$

and

$$
\|f\|_{L^{\infty}\left(H^{k}\right)} \equiv \sup _{0 \leq n \leq N^{*}}\left\|f^{n}\right\|_{k} .
$$

Let $L_{D}^{p}\left(H^{k}\right)=\left\{f:\|f\|_{L^{p}\left(H^{k}\right)}<\infty\right\}, 1 \leq p \leq \infty$. 
We require the following assumptions on the coefficients. Let $k_{*}, k^{*}, \phi_{*}, \phi^{*}$, and $K^{*}$ be positive constants such that

$$
\left.\begin{array}{c}
0<k_{*} \leq \frac{k(\mathbf{x})}{\mu(c)} \leq k^{*}, \quad 0<\phi_{*} \leq \phi(\mathbf{x}) \leq \phi^{*} \\
|\nabla \phi|+\left|\frac{\partial D}{\partial u_{j}}\right|+\left|\frac{\partial \tilde{g}}{\partial t}\right|+\left\|c^{0}\right\|_{s+1} \leq K^{*},
\end{array}\right\}
$$

for some $s \geq 1$. Further assumptions will be made in individual theorems as necessary.

For convenience, we have assumed that $\tilde{q}$ is a smooth function. Under some additional assumptions, our arguments could be modified to include the point source case [6].

\section{Estimates for advection-diffusion problems}

In this section, we assume $u$ in (1.1) is given. Let $h>0$ and $\mathcal{M}_{h}$ be a finite dimensional subspace of $H^{1}(\Omega)$. We assume that there exists an integer $k^{* *}>0$ such that

$$
\begin{gathered}
\inf _{\chi \in \mathcal{M}_{h}}\left[\|f-\chi\|+h\|f-\chi\|_{1}+h\left(\|f-\chi\|_{\infty}+h\|f-\chi\|_{W_{\infty}^{1}}\right)\right] \\
\leq K_{0} h^{l}\|f\|_{l}, \quad 2 \leq l \leq k^{* *}+1, \\
\|\chi\|_{W_{\infty}^{1}} \leq K_{0} h^{-1}\|\chi\|_{1}, \quad\|\chi\|_{\infty} \leq K_{0} h^{-1}\|\chi\|, \\
\|\chi\|_{1} \leq K_{0} h^{-1}\|\chi\|, \quad \chi \in \mathcal{M}_{h},
\end{gathered}
$$

where $K_{0}$ is independent of $h$. It is well known that these properties are valid for continuous piecewise polynomials of degree $\leq k^{* *}$ on a quasi-uniform mesh of diameter $\leq h$.

Our convergence analysis will use a technique based on comparing the finite element approximation to an elliptic projection; see [13]. Define $\tilde{C}(\cdot, t) \in \mathcal{M}_{h}$ by

$$
\begin{gathered}
(D(\mathbf{u}) \nabla \tilde{C}, \nabla \chi)+(\tilde{C}, \chi)+(\tilde{q} \tilde{C}, \chi) \\
=(D(\mathbf{u}) \nabla c, \nabla \chi)+(c, \chi)+(\tilde{q} c, \chi) \\
=-\left(\phi c_{t}, \chi\right)-(\mathbf{u} \cdot \nabla c, \chi)+(c, \chi)+(\tilde{q} \tilde{c}, \chi), \\
\chi \in \mathcal{M}_{h}, \quad t \in J .
\end{gathered}
$$

It is well known that the following elliptic projection results hold; i.e., there exists a constant $K$ such that for $c$ sufficiently smooth and $D(\mathbf{u}) \geq D_{*}>0$,

$$
\begin{gathered}
\|(c-\tilde{C})(t)\| \leq K\left(D_{*}\right) h^{l+1}\|c(\cdot, t)\|_{l+1}, \\
\|\| c-\tilde{C}\left|\left\|_{L^{2}\left(L^{2}\right)}+h D_{*}^{\frac{1}{2}}\right\| c-\tilde{C}\right|\left\|_{L^{2}\left(H^{1}\right)} \leq K h^{l+1}\left|\|c \mid\|_{L^{2}\left(H^{l+1}\right)},\right.\right.
\end{gathered}
$$


and

$$
\left\|(c-\tilde{C})_{t}\right\|_{L^{2}\left(L^{2}\right)} \leq K\left(D_{*}\right) h^{l+1}\|c\|_{H^{1}\left(H^{l+1}\right)}
$$

where $0 \leq l \leq k^{* *}$.

Moreover, in order to obtain a suboptimal estimate for $D(\mathbf{u}) \geq 0$, we let $\tilde{C}$ be the $L^{2}$ projection of $c$, that is

$$
(\phi \tilde{C}, \chi)=(\phi c, \chi), \quad \chi \in \mathcal{M}_{h}
$$

In this case, we have

$$
h\|\| c-\tilde{C}\left\|_{L^{2}\left(H^{1}\right)}^{2}+\right\|\|c-\tilde{C}\|_{L^{2}\left(L^{2}\right)} \leq K h^{l+1}\|\| c \|_{L^{2}\left(H^{l+1}\right)} .
$$

The basic idea of the MMOC is to think of the term

$$
\phi c_{t}+\mathbf{u} \cdot \nabla c
$$

as a directional derivative. Let $\tau$ denote the unit vector in the direction $(\mathbf{u}, \phi)$ in $\Omega \times J$ and set

$$
\theta=\left[|\mathbf{u}|^{2}+(\phi(\mathbf{x}))^{2}\right]^{\frac{1}{2}}
$$

Then one obtains

$$
\theta c_{\tau}-\nabla \cdot D \nabla c=\tilde{q}(\tilde{c}-c)
$$

which has the form of the heat equation. We further note the backward difference approximation

$$
c_{\tau}\left(\mathbf{x}, t^{n}\right) \approx \frac{c\left(\mathbf{x}, t^{n}\right)-c\left(\mathbf{x}-\frac{\mathbf{u}\left(\mathbf{x}, t^{n}\right)}{\phi(\mathbf{x})} \Delta t, t^{n-1}\right)}{\Delta t \sqrt{1+|\mathbf{u}|^{2} / \phi(\mathbf{x})^{2}}}
$$

and if we let

$$
\overline{\mathbf{x}}=\mathbf{x}-\frac{\mathbf{u}\left(\mathbf{x}, t^{n}\right)}{\phi(\mathbf{x})} \Delta t
$$

then

$$
\theta c_{\tau}\left(\mathbf{x}, t^{n}\right) \approx \phi \frac{c\left(\mathbf{x}, t^{n}\right)-c\left(\overline{\mathbf{x}}, t^{n-1}\right)}{\Delta t} .
$$

The modified method of characteristics is a map

$$
C:\left\{t^{0}, t^{1}, \ldots, t^{N^{*}}=T\right\} \rightarrow \mathcal{M}_{h}
$$

defined by

$$
C^{0}(\mathbf{x})=\tilde{C}(\mathbf{x}, 0)
$$


and

$$
\begin{aligned}
\left(\phi \frac{C^{n}-\check{C}^{n-1}}{\Delta t}, \chi\right)+\left(D\left(\mathbf{u}^{n}\right) \nabla C^{n}, \nabla \chi\right)= & \left(\tilde{q}^{n}\left(\tilde{c}^{n}-C^{n}\right), \chi\right), \\
& \chi \in \mathcal{M}_{h}, \quad n \geq 1,
\end{aligned}
$$

where

$$
\check{C}^{n-1}(\mathbf{x})= \begin{cases}C^{n-1}(\check{\mathbf{x}}), & n>1, \\ \tilde{C}(\check{\mathbf{x}}, 0), & n=1 .\end{cases}
$$

In the analysis that follows $K$ will denote a generic positive constant and $\epsilon, \bar{\epsilon}$, and $\delta$ small positive constants, independent of $h$ and $\Delta t$.

Throughout this paper, we will make repeated use of the well-known inequality

$$
a b \leq \frac{\epsilon}{2} a^{2}+\frac{1}{2 \epsilon} b^{2}
$$

which holds for $\epsilon>0, a, b \in \Re$.

\section{1 $L_{D}^{\infty}\left(L^{2}\right)$ estimate}

Set $e^{n}=c^{n}-C^{n}$. Before deriving an error estimate for $e$ in $L_{D}^{\infty}\left(L^{2}\right)$ which assumes only positive-semidefiniteness for $D(\mathrm{u})$, we prove the following lemma. Let

$$
G\left(\mathbf{x}, t^{n}\right)=\check{\mathbf{x}}=\mathbf{x}-\frac{\mathbf{u}\left(\mathbf{x}, t^{n}\right)}{\phi(\mathbf{x})} \Delta t .
$$

We remark that, because of the smoothness and periodicity of $\mathbf{u}$ and $\phi, G$ is a differentiable homeomorphism of $\Omega$ onto itself for $\Delta t$ sufficiently small (see [5]).

Lemma 3.1 Along with (2.1), assume that $\mathbf{u}$ and $\nabla \cdot \frac{\mathbf{u}}{\phi}$ have bounded first partial derivatives in space. Then, for $\Delta t$ sufficiently small, an arbitrary function $f \in L^{2}(\Omega)$ satisfies

$$
\begin{aligned}
& \frac{1}{2 \Delta t}[(\phi \check{f}, \check{f})-(\phi f, f)] \\
& \quad \leq \frac{1}{2}(\tilde{q} f, f)+\epsilon(\phi f, f)+K_{1}(\phi f, f)+K_{2}(f, f),
\end{aligned}
$$

where

$$
\begin{gathered}
K_{1}=K_{1}\left(\|\mathrm{u}\|_{\infty},\left\|\nabla\left(\frac{1}{\phi}\right)\right\|_{\infty}\right), \\
K_{2}=K_{2}\left(\left\|\frac{\mathrm{u}}{\phi}\right\|_{\infty},\|\nabla \phi\|_{\infty}\right) .
\end{gathered}
$$

If $\phi$ is constant, then $K_{1}=K_{2}=0$. 
Proof. For each $\mathbf{x} \in \Omega$, let $\mathbf{y}=G(\mathbf{x}, t) \equiv H(\mathbf{x})$ for some fixed $t \in J, t \geq \Delta t$. The Jacobian of this transformation is

$$
\mathcal{D} H(\mathbf{x})=\left[\begin{array}{cc}
1-\frac{\partial}{\partial x_{1}}\left(\frac{u_{1}}{\phi}\right) \Delta t & -\frac{\partial}{\partial x_{2}}\left(\frac{u_{1}}{\phi}\right) \Delta t \\
-\frac{\partial}{\partial x_{1}}\left(\frac{u_{2}}{\phi}\right) \Delta t & 1-\frac{\partial}{\partial x_{2}}\left(\frac{u_{2}}{\phi}\right) \Delta t
\end{array}\right],
$$

so that the determinant can be expressed as

$$
\operatorname{det} \mathcal{D} H(\mathbf{x})=1-\nabla \cdot \frac{\mathbf{u}}{\phi} \Delta t+\mathcal{O}\left(\Delta t^{2}\right) .
$$

Because $H$ maps the periodic domain $\Omega$ bijectively onto itself, a change of variable yields

$$
\begin{aligned}
(\phi \ddot{f}, \check{f}) & =\int_{\Omega} \phi(\mathbf{x}) f(\mathbf{y}) f(\mathbf{y}) d \mathbf{x} \\
& =\int_{\Omega} \phi(\mathbf{x}) f(\mathbf{y}) f(\mathbf{y}) \frac{1}{\operatorname{det} \mathcal{D} H(\mathbf{x})} d \mathbf{y} \\
& =\int_{\Omega} \phi(\mathbf{x}) f(\mathbf{y}) f(\mathbf{y})\left[1+\left(\nabla \cdot \frac{\mathbf{u}}{\phi}\right)(\mathbf{x}) \Delta t+\mathcal{O}\left(\Delta t^{2}\right)\right] d \mathbf{y}
\end{aligned}
$$

Subtract $(\phi f, f)$ from both sides of (3.19) to obtain

$$
\begin{aligned}
(\phi \check{f}, \check{f})-(\phi f, f) & \\
= & \int_{\Omega}[\phi(\mathbf{x})-\phi(\mathbf{y})] f(\mathbf{y}) f(\mathbf{y})\left[1+\left(\nabla \cdot \frac{\mathbf{u}}{\phi}\right)(\mathbf{x}) \Delta t+\mathcal{O}\left(\Delta t^{2}\right)\right] d \mathbf{y} \\
& +\int_{\Omega} \phi(\mathbf{y}) f(\mathbf{y}) f(\mathbf{y})\left[\left(\nabla \cdot \frac{\mathbf{u}}{\phi}\right)(\mathbf{x}) \Delta t+\mathcal{O}\left(\Delta t^{2}\right)\right] d \mathbf{y} .
\end{aligned}
$$

In the second term on the right-hand side of $(3.20)$, write

$$
\begin{aligned}
\left(\nabla \cdot \frac{\mathbf{u}}{\phi}\right)(\mathbf{x}) & =\left[\left(\nabla \cdot \frac{\mathbf{u}}{\phi}\right)(\mathbf{x})-\left(\nabla \cdot \frac{\mathbf{u}}{\phi}\right)(\mathbf{y})\right]+\frac{1}{\phi}(\nabla \cdot \mathbf{u})(\mathbf{y})+\left(\mathbf{u} \cdot \nabla \frac{1}{\phi}\right)(\mathbf{y}) \\
& =\left[\left(\nabla \cdot \frac{\mathbf{u}}{\phi}\right)(\mathbf{x})-\left(\nabla \cdot \frac{\mathbf{u}}{\phi}\right)(\mathbf{y})\right]+\frac{1}{\phi} q(\mathbf{y})+\left(\mathbf{u} \cdot \nabla \frac{1}{\phi}\right)(\mathbf{y}),
\end{aligned}
$$

and divide by $2 \Delta t$ to see that

$$
\begin{aligned}
\frac{1}{2 \Delta t} & {[(\phi \check{f}, \check{f})-(\phi f, f)] } \\
= & \frac{1}{2 \Delta t} \int_{\Omega}[\phi(\mathbf{x})-\phi(\mathbf{y})] f(\mathbf{y}) f(\mathbf{y})\left[1+\left(\nabla \cdot \frac{\mathbf{u}}{\phi}\right)(\mathbf{x}) \Delta t+\mathcal{O}\left(\Delta t^{2}\right)\right] d \mathbf{y} \\
& +\frac{1}{2} \int_{\Omega} \phi(\mathbf{y}) f(\mathbf{y}) f(\mathbf{y})\left[\left(\nabla \cdot \frac{\mathbf{u}}{\phi}\right)(\mathbf{x})-\left(\nabla \cdot \frac{\mathbf{u}}{\phi}\right)(\mathbf{y})\right] d \mathbf{y}
\end{aligned}
$$




$$
\begin{aligned}
& +\frac{1}{2} \int_{\Omega} q(\mathbf{y}) f(\mathbf{y}) f(\mathbf{y}) d \mathbf{y} \\
& +\frac{1}{2} \int_{\Omega} \phi(\mathbf{y}) f(\mathbf{y}) f(\mathbf{y})\left(\mathbf{u} \cdot \nabla \frac{1}{\phi}\right)(\mathbf{y}) d \mathbf{y} \\
& +\mathcal{O}(\Delta t) \int_{\Omega} \phi(\mathbf{y}) f(\mathbf{y}) f(\mathbf{y}) d \mathbf{y} \\
& \equiv T_{1}+T_{2}+T_{3}+T_{4}+T_{5}
\end{aligned}
$$

We find bounds for the right-hand side of (3.21). In $T_{1}$, note that

$$
|\phi(\mathbf{x})-\phi(\mathbf{y})| \leq\|\nabla \phi\|_{\infty}|\mathbf{x}-\mathbf{y}| \leq\|\nabla \phi\|_{\infty}\left\|\frac{\mathbf{u}}{\phi}\right\|_{\infty} \Delta t
$$

so that

$$
\left|T_{1}\right| \leq\|\nabla \phi\|_{\infty}\left\|\frac{\mathbf{u}}{\phi}\right\|_{\infty}(f, f)=K_{2}(f, f) .
$$

Similarly,

$$
\left|T_{2}\right| \leq \frac{1}{2} \Delta t\left\|\nabla\left(\nabla \cdot \frac{\mathbf{u}}{\phi}\right)\right\|_{\infty}\left\|\frac{\mathbf{u}}{\phi}\right\|_{\infty}(\phi f, f) \leq \epsilon(\phi f, f) .
$$

Next, because $q \leq \tilde{q}=\max (q, 0)$, we have

$$
\left|T_{3}\right| \leq \frac{1}{2}(\tilde{q} f, f)
$$

For the last two terms, we find that

$$
\begin{gathered}
\left|T_{\mathbf{4}}\right| \leq \frac{1}{2}\left\|\mathbf{u} \cdot \nabla\left(\frac{1}{\phi}\right)\right\|_{\infty}(\phi f, f)=K_{1}(\phi f, f), \\
\left|T_{5}\right|=\mathcal{O}(\Delta t)(\phi f, f) \leq \epsilon(\phi f, f) .
\end{gathered}
$$

Putting the above bounds together, the lemma follows. If $\phi$ is constant, an inspection reveals that $T_{1}=T_{4}=0$, so that $K_{1}$ and $K_{2}$ can be zero.

Our basic idea is to compare $C$ with the $L^{2}$ projection $\tilde{C}$ defined by (3.9) and then note that

$$
\left\|\phi^{\frac{1}{2}} e\right\|_{L^{\infty}\left(L^{2}\right)} \leq\|\| \phi^{\frac{1}{2}} \zeta\left\|_{L^{\infty}\left(L^{2}\right)}+\right\|\left\|\phi^{\frac{1}{2}} \xi\right\|_{L^{\infty}\left(L^{2}\right)}
$$

where $\zeta=C-\tilde{C}$, and $\xi=c-\tilde{C}$. 
Subtracting (3.4) from (3.14) and setting $\chi=\zeta^{n}$, we obtain

$$
\begin{gathered}
\left(\phi \frac{\zeta^{n}-\check{\zeta}^{n-1}}{\Delta t}, \zeta^{n}\right)+\left(D\left(\mathbf{u}^{n}\right) \nabla \zeta^{n}, \nabla \zeta^{n}\right)+\left(\tilde{q} \zeta^{n}, \zeta^{n}\right) \\
=\left(\sigma^{n}, \zeta^{n}\right)-\left(\phi \frac{\check{\xi}^{n-1}-\xi^{n-1}}{\Delta t}, \zeta^{n}\right) \\
\quad+\left(D\left(\mathbf{u}^{n}\right) \nabla \xi^{n}, \nabla \zeta^{n}\right)+\left(\tilde{q} \xi^{n}, \zeta^{n}\right)
\end{gathered}
$$

where

$$
\sigma^{n}=\left[\phi c_{t}^{n}+\mathbf{u}^{n} \cdot \nabla c^{n}\right]-\phi \frac{c^{n}-\check{c}^{n-1}}{\Delta t} .
$$

By standard backward difference error analysis [5], we have

$$
\left\|\sigma^{n}\right\|^{2} \leq K \Delta t|| c_{\tau \tau} \|_{L^{2}\left(t^{n-1}, t^{n} ; L^{2}\right)}^{2} .
$$
that

Applying Lemma 3.1 with $f=\zeta^{n-1}$, the Schwartz inequality, and (3.25) we deduce

$$
\begin{aligned}
& \frac{1}{2 \Delta t}\left[\left\|\phi^{\frac{1}{2}} \zeta^{n}\right\|^{2}-\left\|\phi^{\frac{1}{2}} \zeta^{n-1}\right\|^{2}\right]+\left\|D^{\frac{1}{2}}\left(\mathbf{u}^{n}\right) \nabla \zeta^{n}\right\|^{2}+\left\|\tilde{q}^{\frac{1}{2}} \zeta^{n}\right\|^{2} \\
& \leq K \Delta t\left\|c_{\tau \tau}\right\|_{L^{2}\left(t^{n-1}, t^{n} ; L^{2}\right)}^{2}+\left\|\phi^{\frac{1}{2}} \zeta^{n}\right\|^{2}+K\left\|\xi^{n}\right\|^{2} \\
&+K\left\|\phi^{\frac{1}{2}} \frac{\xi^{n-1}-\xi^{n-1}}{\Delta t}\right\|^{2} \\
&+\frac{1}{2}\left\|\tilde{q}^{\frac{1}{2}} \zeta^{n-1}\right\|^{2}+\epsilon\left\|\phi^{\frac{1}{2}} \zeta^{n-1}\right\|^{2} \\
&+K_{1}\left\|\phi^{\frac{1}{2}} \zeta^{n-1}\right\|^{2}+\left(1+K_{2}\right)\left\|\zeta^{n-1}\right\|^{2} \\
&+\frac{1}{2}\left\|D^{\frac{1}{2}}\left(\mathbf{u}^{n}\right) \nabla \zeta^{n}\right\|^{2}+\frac{1}{2}\left\|D^{\frac{1}{2}}\left(\mathbf{u}^{n}\right) \nabla \xi^{n}\right\|^{2},
\end{aligned}
$$

where the functional dependence of $K_{1}$ and $K_{2}$ is described by Lemma 3.1. In particular if $\phi$ is constant then $K_{2}=0$. We observe that by definition of $\check{\xi}$ that

$$
\left\|\phi^{\frac{1}{2}} \frac{\check{\xi}^{n-1}-\xi^{n-1}}{\Delta t}\right\| \leq K\left\|\mathbf{u}^{n} \cdot \nabla \xi^{n-1}\right\| \leq K(\mathrm{u})\left\|\nabla \xi^{n-1}\right\| .
$$

Moreover,

$$
\left\|D^{\frac{1}{2}}\left(\mathbf{u}^{n}\right) \nabla \xi^{n}\right\| \leq K(\mathbf{u})\left\|\nabla \xi^{n}\right\| .
$$

Multiplying (3.26) by $2 \Delta t$, using (3.27) and (3.28), and summing on $n, n=$ $1, \ldots, N$, we see that

$$
\begin{aligned}
\left\|\phi^{\frac{1}{2}} \zeta^{N}\right\|^{2} & +\sum_{n=1}^{N}\left[\left\|D^{\frac{1}{2}}\left(\mathbf{u}^{n}\right) \nabla \zeta^{n}\right\|^{2}+\left\|\tilde{q}^{\frac{1}{2}} \zeta^{n}\right\|^{2}\right] \Delta t \\
\leq & K\left[(\Delta t)^{2}\left\|c_{\tau \tau}\right\|_{L^{2}\left(L^{2}\right)}^{2}+\|\xi\| \|_{L^{2}\left(H^{1}\right)}^{2}\right] \\
& +\sum_{n=1}^{N}\left[\left(2+K_{1}\right)\left\|\phi^{\frac{1}{2}} \zeta^{n}\right\|^{2}+2\left(1+K_{2}\right)\left\|\zeta^{n}\right\|^{2}\right] \Delta t
\end{aligned}
$$


where $K_{1}=K_{2}=0$ if $\phi \equiv$ const. Recall from (3.13) that $\zeta^{0}=0$. Using the bounds for $\phi$ given by $(2.1)$ to write $\left(1+K_{2}\right)\left\|\zeta^{n}\right\|^{2} \leq \frac{1+K_{2}}{\phi_{*}}\left\|\phi^{\frac{1}{2}} \zeta^{n}\right\|^{2}$ and applying Gronwail's Lemma in (3.29), we have

$$
\left\|\phi^{\frac{1}{2}} \zeta\right\|_{L^{\infty}\left(L^{2}\right)}^{2} \leq K\left(\Delta t^{2}+\|\| \xi \|_{L^{2}\left(H^{1}\right)}^{2}\right) .
$$

Thus, by applying the estimate (3.9) for $\xi$, and using (3.22), we have the following theorem.

Theorem 3.1 Let the hypothesis of Lemma 3.1 hold. Assume

$$
c \in H^{2}\left(L^{2}\right) \cap L_{D}^{2}\left(H^{s+1}\right),
$$

for some $s \geq 1$. Then for $\Delta t$ sufficiently small

$$
\left\|\phi^{\frac{1}{2}} e\right\|_{L^{\infty}\left(L^{2}\right)}^{2} \leq K_{3}\left[\Delta t^{2}\left\|c_{\tau \tau}\right\|_{L^{2}\left(L^{2}\right)}^{2}+h^{2 r}\|\| c \|_{L^{2}\left(H^{r+1}\right)}^{2}\right]
$$

where $r=\min \left(k^{* *}, s\right)$ and $K_{3}$ depends on $K_{1}$ and $K_{2}$, among other quantities, but has no dependence on a positive lower bound for $D(\mathbf{u})$. If $\phi$ is constant, then the Gronwall growth in $K_{3}$ depends only on $\phi_{*}=\phi$.

\section{2 $L_{D}^{2}\left(L^{2}\right)$ estimate for $e$}

We now derive an estimate for $\|e\|_{L^{2}\left(L^{2}\right)}$. In this estimate we assume $D$ is positive definite with lower bound $D_{*}>0$. Again set $e^{n}=c^{n}-C^{n}$, then from (1.1) and (3.14) we deduce that

$$
\left(\phi \frac{e^{n}-\check{e}^{n-1}}{\Delta t}, \chi\right)+\left(D\left(\mathbf{u}^{n}\right) \nabla e^{n}, \nabla \chi\right)+\left(\tilde{q} e^{n}, \chi\right)=-\left(\sigma^{n}, \chi\right), \quad \chi \in \mathcal{M}_{h}
$$

where $\sigma^{n}$ is given by (3.24).

We recall that $G\left(\mathbf{x}, t^{n}\right)$ as given by $(3.17)$ is a differentiable homeomorphism of $\Omega$ onto itself. We therefore define

$$
Z^{n}(\mathbf{x})=G^{-1}\left(\mathbf{x}, t^{n}\right)
$$

Let $\breve{g}(\mathbf{x}) \equiv g\left(Z^{n-1}(\mathbf{x})\right)$.

We first need a technical lemma.

Lemma 3.2 Set $\psi^{0}=0$, and for $n=1, \ldots, N^{*}$ define $\psi^{n}$ by

$$
\begin{aligned}
& \frac{\phi \psi^{n}(\mathbf{x})-\breve{\phi} \breve{\psi}^{n-1} \operatorname{det}\left(\mathcal{D} Z^{n-1}(\mathbf{x})\right)}{\Delta t} \\
& -\nabla \cdot \bar{D}\left(\mathbf{u}^{n}\right) \nabla \psi^{n}(\mathbf{x})+\tilde{q} \psi^{n}(\mathbf{x})=\phi e^{n}(\mathbf{x}), \quad \mathbf{x} \in \Omega, \\
& D\left(\mathbf{u}^{n}\right) \nabla \psi^{n} \cdot \eta=0, \quad \mathbf{x} \in \partial \Omega,
\end{aligned}
$$


where $\mathcal{D} Z^{n-1}(\mathbf{x})$ denotes the Jacobian matrix of $Z^{n-1}(\mathbf{x})$. Let the hypothesis of Lemma 3.1 hold, assume $\mathrm{u}_{t}$ exists and is bounded and $D(\mathbf{u})$ is positive definite with lower bound $D_{*}>0$. Then, there exists a constant $K_{4}>0$ such that

$$
\|\| \psi\left\|_{L^{\infty}\left(H^{1}\right)}+\right\|\|\psi\|_{L^{2}\left(H^{2}\right)} \leq K_{4} \mid\left\|\phi^{\frac{1}{2}} e^{n}\right\|_{L^{2}\left(L^{2}\right)} .
$$

Proof. We have for $\chi \in \mathcal{H}^{1}(\Omega)$,

$$
\begin{aligned}
\left(\phi \frac{\psi^{n}-\psi^{n-1}}{\Delta t}\right. & , \chi)+\left(D\left(\mathbf{u}^{n}\right) \nabla \psi^{n}, \nabla \chi\right)+\left(\tilde{q} \psi^{n}, \chi\right)-\left(\phi e^{n}, \chi\right) \\
& =\left(\frac{\breve{\phi} \breve{\psi}^{n-1} \operatorname{det}\left(\mathcal{D} Z^{n-1}\right)-\phi \psi^{n-1}}{\Delta t}, \chi\right) \\
& =\left(\phi \psi^{n-1}, \frac{\chi\left(G^{n-1}\right)-\chi}{\Delta t}\right) .
\end{aligned}
$$

The second step above is obtained by a change of variables argument which again involves the periodicity assumptions made earlier, see [5]. Hence,

$$
\begin{aligned}
& \left(\phi \frac{\psi^{n}-\psi^{n-1}}{\Delta t}, \chi\right)+\left(D\left(\mathbf{u}^{n}\right) \nabla \psi^{n}, \nabla \chi\right) \\
& \quad=-\left(\tilde{q} \psi^{n}, \chi\right)+\left(\phi e^{n}, \chi\right)+\left(\phi \psi^{n-1}, \frac{\chi\left(G^{n-1}\right)-\chi}{\Delta t}\right) \\
& \quad \leq K\left(\left\|\psi^{n}\right\|+\left\|\phi^{\frac{1}{2}} e^{n}\right\|\right)\left\|\phi^{\frac{1}{2}} \chi\right\|+\left\|\phi \psi^{n-1}\right\|_{1}\left\|\frac{\chi\left(G^{n-1}\right)-\chi}{\Delta t}\right\|_{-1} .
\end{aligned}
$$

Following arguments given in [5], we now demonstrate that

$$
\left\|\frac{\chi\left(G^{n-1}\right)-\chi}{\Delta t}\right\|_{-1} \leq K\|\chi\|
$$

Thus, consider

$$
\left\|\frac{\chi\left(G^{n-1}\right)-\chi}{\Delta t}\right\|_{-1}=\sup _{g \in H^{1}(\Omega)}\left[\frac{1}{\|g\|_{1}} \int_{\Omega} \frac{\chi\left(G^{n-1}(\mathbf{x})\right)-\chi(\mathbf{x})}{\Delta t} g(\mathbf{x}) d \mathbf{x}\right] .
$$

By a change of variables we have

$$
\begin{aligned}
\int_{\Omega} \frac{\chi\left(G^{n-1}(\mathbf{x})\right)-\chi(\mathbf{x})}{\Delta t} g(\mathbf{x}) d \mathbf{x} \\
=\frac{1}{\Delta t}\left[\int_{\Omega} \chi(\mathbf{x}) \breve{g}(\mathbf{x}) \operatorname{det} \mathcal{D} Z^{n-1}(\mathbf{x}) d \mathbf{x}-\int_{\Omega} \chi(\mathbf{x}) g(\mathbf{x}) d \mathbf{x}\right] \\
=\frac{1}{\Delta t}\left[\int_{\Omega} \chi(\mathbf{x}) g(\mathbf{x})\left(\operatorname{det} \mathcal{D} Z^{n-1}(\mathbf{x})-1\right) d \mathbf{x}\right. \\
\left.\quad \quad \quad \int_{\Omega} \chi(\mathbf{x})\left(g\left(Z^{n-1}(\mathbf{x})\right)-g(\mathbf{x})\right) \operatorname{det} \mathcal{D} Z^{n-1}(\mathbf{x}) d \mathbf{x}\right]
\end{aligned}
$$


Thus

$$
\begin{aligned}
& \left\|\frac{\chi\left(G^{n-1}\right)-\chi}{\Delta t}\right\|_{-1} \\
& \leq \frac{1}{\Delta t} \sup _{g \in H^{1}(\Omega)}\left[\frac{1}{\|g\|_{1}} \int_{\Omega} \chi(\mathbf{x}) g(\mathbf{x})\left(\operatorname{det} \mathcal{D} Z^{n-1}(\mathbf{x})-1\right) d \mathbf{x}\right] \\
& \quad+\frac{1}{\Delta t} \sup _{g \in H^{1}(\Omega)}\left[\frac{1}{\|g\|_{1}} \int_{\Omega} \chi(\mathbf{x})\left(g\left(Z^{n-1}(\mathbf{x})\right)-g(\mathbf{x})\right) \operatorname{det} \mathcal{D} Z^{n-1}(\mathbf{x}) d \mathbf{x}\right] \\
& \equiv W_{1}+W_{2} .
\end{aligned}
$$

By (3.17), (3.32), and (3.18) and the assumptions on $\mathbf{u}$ and $\phi$ there exists a constant $K_{5}$ such that

$$
\left|\operatorname{det} \mathcal{D} Z^{n-1}(\mathbf{x})-1\right| \leq\left\|\nabla \cdot \frac{\mathrm{u}}{\phi}\right\|_{\infty} \Delta t+\mathcal{O}\left(\Delta t^{2}\right) \leq K_{5} \Delta t
$$

and

$$
\left|\mathbf{x}-Z^{n-1}(\mathbf{x})\right| \leq\left\|\frac{\mathbf{u}}{\phi}\right\|_{\infty} \Delta t \leq K_{5} \Delta t
$$

Hence,

$$
\left|W_{1}\right| \leq K_{5} \sup _{g \in H^{1}}\left[\|\chi\|\|g\| /\|g\|_{1}\right] \leq K_{5}\|\chi\|
$$

Next, let $\mathbf{z}$ be the unit vector in the direction of $\mathbf{x}-Z^{n-1}(\mathbf{x})$, and let $\bar{z} \in[0,1]$ parametrize the line segment from $x$ to $Z^{n-1}(x)$. Then

$$
\left|W_{2}\right| \leq \frac{K}{\Delta t} \sup _{g \in H^{1}}\left[\frac{1}{\|g\|_{1}} \int_{\Omega} \chi(\mathbf{x}) \tilde{g}(\mathbf{x})\left|\mathbf{x}-Z^{n-1}(\mathbf{x})\right| d \mathbf{x}\right]
$$

where

$$
\tilde{g}(\mathbf{x})=\int_{0}^{1} \frac{\partial g}{\partial \mathbf{z}}\left((1-\bar{z}) Z^{n-1}(\mathbf{x})+\bar{z} \mathbf{x}\right) d \bar{z}
$$

Moreover, since $Z^{n-1}(\mathbf{x})$ is continuous and differentiable, we have

$$
\|\tilde{g}\| \leq K\|\nabla g\|
$$

Thus, combining (3.42)-(3.43) and (3.40) we find

$$
\left|W_{2}\right| \leq K K_{5}\|\chi\|
$$

and (3.37) now follows by combining (3.38), (3.41), and (3.44). 
Returning now to $(3.36)$, let $\chi=\left(\psi^{n}-\psi^{n-1}\right) / \Delta t$, then manipulating and applying (3.37) we obtain

$$
\begin{aligned}
& \left\|\phi^{\frac{1}{2}} \frac{\psi^{n}-\psi^{n-1}}{\Delta t}\right\|^{2}+\frac{1}{2 \Delta t}\left[\left\|D^{\frac{1}{2}}\left(\mathbf{u}^{n}\right) \nabla \psi^{n}\right\|^{2}-\left\|D^{\frac{1}{2}}\left(\mathbf{u}^{n-1}\right) \nabla \psi^{n-1}\right\|^{2}\right] \\
& \leq K\left\|\phi^{\frac{1}{2}} e^{n}\right\|^{2}+K\left\|\psi^{n}\right\|_{1}^{2}+K K_{5}^{2}\left\|\psi^{n-1}\right\|_{1}^{2}+\frac{1}{2}\left\|\phi^{\frac{1}{2}} \frac{\psi^{n}-\psi^{n-1}}{\Delta t}\right\|^{2} \\
& \quad+\frac{1}{2 \Delta t}\left(\left[D^{\frac{1}{2}}\left(\mathbf{u}^{n}\right)-D^{\frac{1}{2}}\left(\mathbf{u}^{n-1}\right)\right] \nabla \psi^{n-1}, \nabla \psi^{n-1}\right) \\
& \leq K\left(K_{5},\left\|\mathbf{u}_{t}\right\|_{L^{\infty}\left(L^{\infty}\right)}\right)\left\|\psi^{n-1}\right\|_{1}^{2}+K\left\|\psi^{n}\right\|_{1}^{2}+K\left\|\phi^{\frac{1}{2}} e^{n}\right\|^{2}+\frac{1}{2}\left\|\phi^{\frac{1}{2}} \frac{\psi^{n}-\psi^{n-1}}{\Delta t}\right\|^{2} \\
& \leq K_{6}\left(\left\|\psi^{n-1}\right\|_{1}^{2}+\left\|\psi^{n}\right\|_{1}^{2}\right)+K\left\|\phi^{\frac{1}{2}} e^{n}\right\|^{2}+\frac{1}{2}\left\|\phi^{\frac{1}{2}} \frac{\psi^{n}-\psi^{n-1}}{\Delta t}\right\|^{2} .
\end{aligned}
$$

Multiplying (3.45) by $\Delta t$, summing on $n$ and applying the discrete Gronwall Lemma we find

$$
\sum_{n=1}^{N^{*}}\left\|\phi^{\frac{1}{2}} \frac{\psi^{n}-\psi^{n-1}}{\Delta t}\right\|^{2} \Delta t+\|\| \psi\left\|_{L^{\infty}\left(H^{1}\right)}^{2} \leq K_{7}\right\| \phi^{\frac{1}{2}} e^{n}\|\|_{L^{2}\left(L^{2}\right)}^{2}
$$

where

$$
K_{7}=K_{7}\left(K_{5},\left\|\mathbf{u}_{t}\right\|_{L^{\infty}\left(L^{\infty}\right)},\left(D_{*}\right)^{-1}\right)
$$

For an $H^{2}$ estimate of $\psi$, rewrite $(3.33)$ as

$$
\begin{aligned}
& -\nabla \cdot\left(D\left(\mathbf{u}^{n}\right) \nabla \psi^{n}(\mathbf{x})\right)=\phi e^{n}(\mathbf{x})-\tilde{q} \psi^{n}(\mathbf{x}) \\
& -\frac{\phi(\mathbf{x}) \psi^{n}(\mathbf{x})-\breve{\phi}(\mathbf{x}) \breve{\psi}^{n-1}(\mathbf{x}) \operatorname{det} \mathcal{D} Z^{n-1}(\mathbf{x})}{\Delta t}
\end{aligned}
$$

From elliptic regularity there exists a constant $K_{8}=K_{8}\left(D_{*}, \Omega\right)$ such that

$$
\begin{aligned}
\left\|\psi^{n}\right\|_{2}^{2} \leq K_{8}[ & \left\|\phi \frac{\psi^{n}-\psi^{n-1}}{\Delta t}\right\|^{2}+\left\|\phi^{\frac{1}{2}} e^{n}\right\|^{2}+\left\|\tilde{q} \psi^{n}\right\|^{2} \\
& \left.+\left\|\frac{\phi \psi^{n-1}-\check{\phi} \breve{\psi}^{n-1} \operatorname{det} \mathcal{D} Z^{n-1}}{\Delta t}\right\|^{2}\right] .
\end{aligned}
$$

Consider the last term on the right-hand side of (3.48). We have

$$
\begin{aligned}
& \frac{\phi(\mathbf{x}) \psi^{n-1}(\mathbf{x})-\breve{\phi}(\mathbf{x}) \breve{\psi}^{n-1}(\mathbf{x}) \operatorname{det} \mathcal{D} Z^{n-1}(\mathbf{x})}{\Delta t} \\
& =\frac{1}{\Delta t}\left[\phi(\mathbf{x}) \psi^{n-1}(\mathbf{x})\left(1-\operatorname{det} \mathcal{D} Z^{n-1}(\mathbf{x})\right)\right. \\
& \left.\quad+\operatorname{det} \mathcal{D} Z^{n-1}(\mathbf{x})\left(\phi(\mathbf{x}) \psi^{n-1}(\mathbf{x})-\breve{\phi}(\mathbf{x}) \breve{\psi}^{n-1}(\mathbf{x})\right)\right] .
\end{aligned}
$$


Thus

$$
\begin{aligned}
\left\|\frac{\phi \psi^{n}-\breve{\phi} \breve{\psi}^{n-1} \operatorname{det} \mathcal{D} Z^{n-1}}{\Delta t}\right\| \\
\leq \frac{1}{\Delta t}\left\|\phi \psi^{n-1}\left(1-\operatorname{det} \mathcal{D} Z^{n-1}\right)\right\| \\
\quad+\frac{1}{\Delta t}\left\|\left(\phi \psi^{n-1}-\breve{\phi} \breve{\psi}^{n-1}\right) \operatorname{det} \mathcal{D} Z^{n-1}\right\| \\
\equiv W_{3}+W_{4} .
\end{aligned}
$$

By (3.39), we have

$$
W_{3} \leq K K_{5}\left\|\psi^{n-1}\right\|
$$

Furthermore, by essentially the same argument which led to (3.44) we obtain

$$
\begin{aligned}
W_{4} & \leq K K_{5}\left\|\phi \psi^{n-1}\right\|_{1} \\
& \leq K K_{5}\left\|\psi^{n-1}\right\|_{1} .
\end{aligned}
$$

Substituting (3.49)-(3.51) in (3.48) we find

$$
\left\|\psi^{n}\right\|_{2}^{2} \leq K\left(K_{8}, K_{5}\right)\left(\left\|\phi^{\frac{1}{2}} e^{n}\right\|^{2}+\left\|\psi^{n-1}\right\|_{1}^{2}+\left\|\psi^{n}\right\|_{1}^{2}+\left\|\phi^{\frac{1}{2}} \frac{\psi^{n}-\psi^{n-1}}{\Delta t}\right\|^{2}\right)
$$

Multiplying above by $\Delta t$, summing on $n$, and applying (3.46), we obtain (3.35). Note that

$$
K_{4}=K_{4}\left(K_{5}, K_{7}, K_{8}\right),
$$

where $K_{5}$ is given by (3.39) and (3.40), $K_{7}$ by $(3.47)$, and $K_{8}$ by (3.48).

We now derive an estimate for $\left\|\phi^{\frac{1}{2}} e\right\|_{L^{2}\left(L^{2}\right)}$, utilizing a parabolic lift argument similar to one developed by Palmer in [8] for a Galerkin approximation of the contaminant transport problem.

Let $\psi^{N^{*}+1}=0$, and for $n=0, \ldots, N^{*}$, let $\psi^{n}$ satisfy the 'backward' equation

$$
\begin{gathered}
\frac{\phi(\mathbf{x}) \psi^{n}(\mathbf{x})-\phi\left(Z^{n+1}(\mathbf{x})\right) \psi^{n+1}\left(Z^{n+1}(\mathbf{x})\right) \operatorname{det} \mathcal{D} Z^{n+1}(\mathbf{x})}{\Delta t} \\
-\nabla \cdot\left(D\left(\mathbf{u}^{n}\right) \nabla \psi^{n}(\mathbf{x})\right)+\tilde{q} \psi^{n}(\mathbf{x})=\phi e^{n}(\mathbf{x}), \quad \mathbf{x} \in \Omega \\
D\left(\mathbf{u}^{n}\right) \nabla \psi^{n} \cdot \eta=0, \quad \mathbf{x} \in \partial \Omega
\end{gathered}
$$

Then, by the lemma just proved applied to $\gamma^{n}=\psi^{N^{*}+1-n}$, we have a bound for $\||\psi|\|_{L^{\infty}\left(H^{1}\right)}+\||| \psi \mid\|_{L^{2}\left(H^{2}\right)}$ of the form (3.35). 
Multiplying (3.53) by $e^{n}(\mathbf{x})$, integrating over $\Omega$, multiplying by $\Delta t$ and summing on $n$ we obtain

$$
\begin{aligned}
\left\|\phi^{\frac{1}{2}} e\right\|_{L^{2}\left(L^{2}\right)}^{2}= & \sum_{n=1}^{N^{*}}\left(\frac{\phi \psi^{n}-\phi\left(Z^{n+1}\right) \psi^{n+1}\left(Z^{n+1}\right) \operatorname{det} \mathcal{D} Z^{n+1}}{\Delta t}, e^{n}\right) \Delta t \\
& +\sum_{n=1}^{N^{*}}\left(D\left(\mathbf{u}^{n}\right) \nabla \psi^{n}, \nabla e^{n}\right) \Delta t+\sum_{n=1}^{N^{*}}\left(\tilde{q} \psi^{n}, e^{n}\right) \Delta t
\end{aligned}
$$

Consider the first term on the right-hand side of (3.55). We want to show this term is equal to

$$
\sum_{n=1}^{N^{*}}\left(\phi \frac{e^{n}-\check{e}^{n-1}}{\Delta t}, \psi^{n}\right) \Delta t+\left(\phi \tilde{e}^{0}, \psi^{1}\right) .
$$

Thus

$$
\begin{aligned}
\sum_{n=1}^{N^{*}} & \left(\phi \frac{e^{n}-\check{e}^{n-1}}{\Delta t}, \psi^{n}\right) \Delta t \\
= & \frac{1}{\Delta t} \sum_{n=1}^{N^{*}}\left[\int_{\Omega} \phi(\mathbf{x}) e^{n}(\mathbf{x}) \psi^{n}(\mathbf{x}) d \mathbf{x}-\int_{\Omega} \phi(\mathbf{x}) e^{n-1}\left(G\left(\mathbf{x}, t^{n}\right)\right) \psi^{n}(\mathbf{x}) d \mathbf{x}\right] \Delta t \\
= & \frac{1}{\Delta t} \sum_{n=1}^{N^{*}}\left[\int_{\Omega} \phi(\mathbf{x}) e^{n}(\mathbf{x}) \psi^{n}(\mathbf{x}) d \mathbf{x}-\int_{\Omega} \phi(\mathbf{x}) e^{n}\left(G\left(\mathbf{x}, t^{n+1}\right)\right) \psi^{n+1}(\mathbf{x}) d \mathbf{x}\right] \Delta t \\
\quad & \quad-\left(\phi \check{e}^{\mathbf{0}}, \psi^{1}\right) \\
= & \sum_{n=1}^{N^{*}}\left(e^{n}, \frac{\phi \psi^{n}-\phi\left(Z^{n+1}\right) \psi^{n+1}\left(Z^{n+1}\right) \operatorname{det} \mathcal{D} Z^{n+1}}{\Delta t}\right) \Delta t \\
& \quad-\left(\phi \ddot{e}^{\mathbf{0}}, \psi^{1}\right),
\end{aligned}
$$

where, in the second step above, we utilized the fact that $\psi^{N^{*}+1}=0$.

Hence, by (3.55), (3.56), and (3.31), we have

$$
\begin{aligned}
& \left\|\phi^{\frac{1}{2}} e\right\|_{L^{2}\left(L^{2}\right)}^{2}=\sum_{n=1}^{N^{*}}\left(\phi \frac{e^{n}-\check{e}^{n}}{\Delta t}, \psi^{n}\right) \Delta t+\sum_{n=1}^{N^{*}}\left(D\left(\mathbf{u}^{n}\right) \nabla e^{n}, \nabla \psi^{n}\right) \Delta t \\
& +\sum_{n=1}^{N^{*}}\left(\tilde{q} e^{n}, \psi^{n}\right) \Delta t+\left(\phi \tilde{e}^{0}, \psi^{1}\right) \\
& =\sum_{n=1}^{N^{*}}\left(\phi \frac{e^{n}-e^{n-1}}{\Delta t}, \psi^{n}-\chi^{n}\right) \Delta t+\left(\phi \check{e}^{0}, \psi^{1}\right) \\
& +\sum_{n=1}^{N^{*}}\left(\phi \frac{e^{n-1}-\dot{e}^{n-1}}{\Delta t}, \psi^{n}-\chi^{n}\right) \Delta t \\
& +\sum_{n=1}^{N^{*}}\left(D\left(\mathbf{u}^{n}\right) \nabla e^{n}, \nabla\left(\psi^{n}-\chi^{n}\right)\right) \Delta t \\
& +\sum_{n=1}^{N^{*}}\left(\tilde{q} e^{n}, \psi^{n}-\chi^{n}\right) \Delta t-\sum_{n=1}^{N^{*}}\left(\sigma^{n}, \chi^{n}\right) \Delta t, \chi^{n} \in \mathcal{M}_{h}
\end{aligned}
$$


Let $\chi^{n}=\psi_{I}^{n}$, where $\psi_{I}^{n}$ is the interpolant of $\psi^{n}$ in $\mathcal{M}_{h}$, then from approximation theory there exists a constant $K_{9}$ such that

$$
\left\|\psi^{n}-\psi_{I}^{n}\right\|_{l} \leq K_{9} h^{2-l}\left\|\psi^{n}\right\|_{2-l}, \quad 0 \leq l \leq 2 .
$$

Thus, by (3.57), (3.58), and (3.35), and applying negative norm estimates to the second term in (3.57), we obtain

$$
\begin{aligned}
\left\|\phi^{\frac{1}{2}} e\right\| \|_{L^{2}\left(L^{2}\right)}^{2} & \\
\leq & K K_{4} K_{9} h^{4} \sum_{n=1}^{N^{*}}\left\|\frac{e^{n}-e^{n-1}}{\Delta t}\right\|^{2} \Delta t+K K_{4} K_{9} h^{2}\left\|D^{\frac{1}{2}}\left(\mathbf{u}^{n}\right) \nabla e^{n}\right\| \|_{L^{2}\left(L^{2}\right)}^{2} \\
& +K K_{4} K_{5} K_{9} h^{2}\left\|\phi^{\frac{1}{2}} e\right\|\left\|_{L^{\infty}\left(L^{2}\right)}^{2}+K K_{4}\right\| e^{0} \|^{2} \\
& +K K_{9}\|\boldsymbol{\sigma}\|\left\|_{L^{2}\left(L^{2}\right)}^{2}+\frac{1}{2}\right\| \phi^{\frac{1}{2} e} e \|_{L^{2}\left(L^{2}\right)}^{2} .
\end{aligned}
$$

Combining (3.59) and (3.25), we have an estimate for $\left\|\phi^{\frac{1}{2}} e\right\|_{L^{2}\left(L^{2}\right)}$, given in the following theorem.

Theorem 3.2 . Assume the hypothesis of Lemma 3.2 holds. Then, there exists a constant $K_{10}$ such that

$$
\begin{aligned}
& \left\|\phi^{\frac{1}{2}} e\right\|_{L^{2}\left(L^{2}\right)} \leq K_{10}\left\{\Delta t+h^{2}\left(\sum_{n=1}^{N^{*}}\left\|\frac{e^{n}-e^{n-1}}{\Delta t}\right\|^{2} \Delta t\right)^{\frac{1}{2}}+h\left\|\phi^{\frac{1}{2}} e\right\|_{L^{\infty}\left(L^{2}\right)}\right. \\
& \left.+h\|\| D^{\frac{1}{2}}(\mathbf{u}) \nabla e\|\|_{L^{2}\left(L^{2}\right)}+\left\|e^{0}\right\|\right\},
\end{aligned}
$$

where $K_{10}=K_{10}\left(K_{9}, K_{5}, K_{4},\left\|c_{\tau \tau}\right\|_{L^{2}\left(L^{2}\right)}\right)$.

We now derive estimates for the terms on the right-hand side of (3.60). We compare $C$ with the elliptic projection $\tilde{C}$ defined by (3.4).

Let $\partial_{t} f^{n} \equiv\left(f^{n}-f^{n-1}\right) / \Delta t$. Recall, by subtracting (3.4) from (3.14) and setting $\zeta=C-\tilde{C}$ and $\xi=c-\tilde{C}$, we obtain

$$
\begin{aligned}
& \left(\phi \partial_{t} \zeta^{n}, \chi\right)+\left(D\left(\mathbf{u}^{n}\right) \nabla \zeta^{n}, \nabla \chi\right) \\
& \quad=\left(\sigma^{n}, \chi\right)+\left(\phi \partial_{t} \xi^{n}, \chi\right)-\left(\xi^{n}, \chi\right)-\left(\tilde{q}^{n} \zeta^{n}, \chi\right) \\
& \quad-\left(\phi \frac{\check{\xi}^{n-1}-\xi^{n-1}}{\Delta t}, \chi\right)+\left(\phi \frac{\check{\zeta}^{n-1}-\zeta^{n-1}}{\Delta t}, \chi\right), \quad \chi \in \mathcal{M}_{h},
\end{aligned}
$$

and we note that

$$
\begin{aligned}
& \left\|\phi_{\phi^{\frac{1}{2}} e}\right\|\left\|_{L^{\infty}\left(L^{2}\right)}+\right\|\left\|D^{\frac{1}{2}}(\mathbf{u}) \nabla e\right\|_{L^{2}\left(L^{2}\right)}+\left\|\partial_{t} e\right\|_{L^{2}\left(L^{2}\right)} \\
& \leq\left.\quad\|\| \phi^{\frac{1}{2}} \zeta\left\|_{L^{\infty}\left(L^{2}\right)}+\right\| D^{\frac{1}{2}}(\mathbf{u}) \nabla \zeta\left\|_{L^{2}\left(L^{2}\right)}+\right\|\right|_{t} \zeta^{n} \|_{L^{2}\left(L^{2}\right)} \\
& \quad+\|\| \phi^{\frac{1}{2}} \xi\left\|_{L^{\infty}\left(L^{2}\right)}+\right\| \mid D^{\frac{1}{2}}(\mathbf{u}) \nabla \xi\left\|_{L^{2}\left(L^{2}\right)}+\right\|\left\|\partial_{t} \xi^{n}\right\| \|_{L^{2}\left(L^{2}\right)} .
\end{aligned}
$$


Setting $\chi=\zeta^{n}$ in (3.61), we have

$$
\begin{aligned}
\left(\phi \partial_{t} \zeta^{n}, \zeta^{n}\right)+\left(D\left(\mathbf{u}^{n}\right) \nabla \zeta^{n}, \nabla \zeta^{n}\right) & \\
= & \left(\sigma^{n}, \zeta^{n}\right)+\left(\phi \partial_{t} \xi^{n}, \zeta^{n}\right)-\left(\xi^{n}, \zeta^{n}\right)-\left(\tilde{q}^{n} \zeta^{n}, \zeta^{n}\right) \\
& -\left(\phi \frac{\check{\xi}^{n-1}-\xi^{n-1}}{\Delta t}, \zeta^{n}\right)+\left(\phi \frac{\check{\zeta}^{n-1}-\zeta^{n-1}}{\Delta t}, \zeta^{n}\right) .
\end{aligned}
$$

Thus, applying the inequality $b(b-c) \geq \frac{1}{2}\left(b^{2}-c^{2}\right)$ and manipulating (3.63) we obtain

$$
\begin{aligned}
\frac{1}{2 \Delta t}[ & \left.\left\|\phi^{\frac{1}{2}} \zeta^{n}\right\|^{2}-\left\|\phi^{\frac{1}{2}} \zeta^{n-1}\right\|^{2}\right]+\left\|D^{\frac{1}{2}}\left(\mathbf{u}^{n}\right) \nabla \zeta^{n}\right\|^{2} \\
\leq & K\left\|\sigma^{n}\right\|^{2}+K\left(\left\|\phi^{\frac{1}{2}} \zeta^{n}\right\|^{2}+\left\|\xi^{n}\right\|^{2}+\left\|\partial_{t} \xi\right\|\left\|\phi^{\frac{1}{2}} \zeta^{n}\right\|\right) \\
& +\left\|\frac{\xi^{n-1}-\xi^{n-1}}{\Delta t}\right\|_{-1}\left\|\phi \zeta^{n}\right\|_{1}+\left\|\frac{\zeta^{n-1}-\zeta^{n-1}}{\Delta t}\right\|-1\left\|\phi \zeta^{n}\right\|_{1} .
\end{aligned}
$$

Using the time difference estimate for $\xi$ derived in [4], we have that

$$
\left\|\partial_{t} \xi^{n}\right\|\left\|\phi^{\frac{1}{2}} \zeta^{n}\right\| \leq K(\Delta t)^{-1} h^{2}\left\|\xi_{t}\right\|_{L^{2}\left(t^{n-1}, t^{n} ; L^{2}\right)}^{2}+\frac{\epsilon h^{-2}}{3}\left\|\phi^{\frac{1}{2}} \zeta^{n}\right\|^{2} .
$$

Also, by (3.37) and inverse assumptions (3.3) we note that

$$
\left\|\frac{\xi^{n-1}-\xi^{n-1}}{\Delta t}\right\|_{-1}\left\|\phi \zeta^{n}\right\|_{1} \leq K K_{0}^{2}\left\|\xi^{n-1}\right\|^{2}+\frac{\epsilon h^{-2}}{3}\left\|\phi^{\frac{1}{2}} \zeta^{n}\right\|^{2}
$$

and

$$
\left\|\frac{\check{\zeta}^{n-1}-\zeta^{n-1}}{\Delta t}\right\|_{-1}\left\|\phi \zeta^{n}\right\|_{1} \leq K K_{0}^{2}\left\|\phi^{\frac{1}{2}} \zeta^{n-1}\right\|^{2}+\frac{\epsilon h^{-2}}{3}\left\|\phi^{\frac{1}{2}} \zeta^{n}\right\|^{2} .
$$

Substituting (3.25), (3.65)-(3.67) into (3.64), we deduce that

$$
\begin{aligned}
& \frac{1}{2 \Delta t}\left[\left\|\phi^{\frac{1}{2}} \zeta^{n}\right\|^{2}-\left\|\phi^{\frac{1}{2}} \zeta^{n-1}\right\|^{2}\right]+\left\|D^{\frac{1}{2}}\left(\mathbf{u}^{n}\right) \nabla \zeta^{n}\right\|^{2} \\
& \leq K\left(\left\|c_{\tau \tau}\right\|_{L^{2}\left(t^{n-1}, t^{n} ; L^{2}\right)}^{2} \Delta t+\left\|\phi^{\frac{1}{2}} \zeta^{n}\right\|^{2}+\left\|\xi^{n}\right\|^{2}+\left\|\phi^{\frac{1}{2}} \zeta^{n-1}\right\|^{2}+\left\|\xi^{n-1}\right\|^{2}\right. \\
& \left.\quad+(\Delta t)^{-1} h^{2}\left\|\xi_{t}\right\|_{L^{2}\left(t^{n-1}, t^{n} ; L^{2}\right)}^{2}\right)+\epsilon h^{-2}\left\|\phi^{\frac{1}{2}} \zeta^{n}\right\|^{2}
\end{aligned}
$$

Multiplying (3.68) by $2 \Delta t$ and summing on $n$, we obtain

$$
\begin{aligned}
\left\|\phi^{\frac{1}{2}} \zeta^{N}\right\|^{2} & +\sum_{n=1}^{N}\left\|D^{\frac{1}{2}}\left(\mathbf{u}^{n}\right) \nabla \zeta^{n}\right\|^{2} \Delta t \\
\leq K & \left(\left\|c_{\tau \tau}\right\|_{L^{2}\left(L^{2}\right)}^{2} \Delta t^{2}+\|\| \xi\left\|_{L^{2}\left(L^{2}\right)}^{2}+h^{2}\right\| \xi_{t} \|_{L^{2}\left(L^{2}\right)}^{2}\right) \\
& +\left(K+\epsilon h^{-2}\right)\left\|\phi^{\frac{1}{2}} \zeta\right\|_{L^{2}\left(L^{2}\right)}^{2} .
\end{aligned}
$$


Noting above that $N$ is arbitrary, multiplying (3.69) by $h^{2}$ and substituting (3.7) and (3.8) into (3.69) we see that

$$
\begin{aligned}
h^{2}\left\|\phi^{\frac{1}{2}} \zeta\right\|_{L^{\infty}\left(L^{2}\right)}^{2}+h^{2} \mid\left\|D^{\frac{1}{2}}(\mathbf{u}) \nabla \zeta\right\|_{L^{2}\left(L^{2}\right)}^{2} \\
\leq K h^{2}(\Delta t)^{2}\left\|c_{\tau \tau}\right\|_{L^{2}\left(L^{2}\right)}^{2}+\left(K h^{2}+\epsilon\right)\left\|\mid \phi^{\frac{1}{2}} e\right\| \|_{L^{2}\left(L^{2}\right)}^{2} \\
\quad+K h^{2(r+1)}\left[\left(h^{2}+\epsilon\right)\left\|c\left|\left\|_{L^{2}\left(H^{r+1}\right)}^{2}+\right\|\right|\right\| c \|_{H^{1}\left(H^{r-1}\right)}^{2}\right],
\end{aligned}
$$

where $r=\min \left(k^{* *}, s\right)$, and $s$ involves smoothness assumptions on $c$ and is defined below.

We now estimate $\left\|\mid \partial_{t} e\right\|_{L^{2}\left(L^{2}\right)}$. In (3.61), setting $\chi=\partial_{t} \zeta^{n}$, we obtain

$$
\begin{aligned}
\left(\phi \partial_{t} \zeta^{n}, \partial_{t} \zeta^{n}\right)+\left(D\left(\mathbf{u}^{n}\right) \nabla \zeta^{n}, \nabla \partial_{t} \zeta^{n}\right) & \\
= & \left(\sigma^{n}, \partial_{t} \zeta^{n}\right)+\left(\phi \partial_{t} \xi^{n}, \partial_{t} \zeta^{n}\right)-\left(\xi^{n}, \partial_{t} \zeta^{n}\right)-\left(\tilde{q}^{n} \zeta^{n}, \partial_{t} \zeta^{n}\right) \\
& -\left(\phi \frac{\xi^{n-1}-\xi^{n-1}}{\Delta t}, \partial_{t} \zeta^{n}\right)+\left(\phi \frac{\zeta^{n-1}-\zeta^{n-1}}{\Delta t}, \partial_{t} \zeta^{n}\right) .
\end{aligned}
$$

Thus

$$
\begin{aligned}
& \left\|\phi^{\frac{1}{2}} \partial_{t} \zeta^{n}\right\|^{2}+\left(D\left(\mathbf{u}^{n}\right) \nabla \zeta^{n}, \nabla \partial_{t} \zeta^{n}\right) \\
& \leq\left\|\sigma^{n}\right\|\left\|\partial_{t} \zeta^{n}\right\|+K\left\|\partial_{t} \xi^{n}\right\|\left\|\partial_{t} \zeta^{n}\right\|+\left\|\xi^{n}\right\|\left\|\partial_{t} \zeta^{n}\right\|+\|\tilde{q}\|\left\|_{L^{\infty}\left(L^{\infty}\right)}\right\| \zeta^{n}\|\| \partial_{t} \zeta^{n} \| \\
& \quad+K\left\|\partial_{t} \zeta^{n}\right\|_{1}\left(\left\|\frac{\xi^{\xi^{n-1}}-\xi^{n-1}}{\Delta t}\right\|_{-1}+\left\|\frac{\zeta^{n-1}-\zeta^{n-1}}{\Delta t}\right\|_{-1}\right) .
\end{aligned}
$$

Now

$$
\begin{aligned}
& \left(D\left(\mathbf{u}^{n}\right) \nabla \zeta^{n}, \nabla \partial_{t} \zeta^{n}\right) \\
& \equiv \frac{1}{\Delta t}\left(\left(D\left(\mathbf{u}^{n}\right) \nabla \zeta^{n}, \nabla \zeta^{n}\right)-\left(D\left(\mathbf{u}^{n}\right) \nabla \zeta^{n}, \nabla \zeta^{n-1}\right)\right. \\
& =\frac{1}{\Delta t}\left[\left(D\left(\mathbf{u}^{n}\right) \nabla \zeta^{n}, \nabla \zeta^{n}\right)-\left(D\left(\mathbf{u}^{n-1}\right) \nabla \zeta^{n-1}, \nabla \zeta^{n-1}\right)\right] \\
& \quad-\left(\partial_{t} D\left(\mathbf{u}^{n}\right) \nabla \zeta^{n-1}, \nabla \zeta^{n-1}\right)+\Delta t\left(D\left(\mathbf{u}^{n}\right) \nabla \partial_{t} \zeta^{n}, \nabla \partial_{t} \zeta^{n}\right) \\
& \quad-\left(D\left(\mathbf{u}^{n}\right) \nabla \partial_{t} \zeta^{n}, \nabla \zeta^{n}\right) .
\end{aligned}
$$

We use inverse assumptions to treat the following terms; that is

$$
\left(\partial_{t} D\left(\mathbf{u}^{n}\right) \nabla \zeta^{n-1}, \nabla \zeta^{n-1}\right) \leq K K_{0}^{2} h^{-2}\left\|\mathbf{u}_{t}\right\|_{\infty}\left\|\zeta^{n-1}\right\|^{2}
$$

and

$$
\left(D\left(\mathbf{u}^{n}\right) \nabla \partial_{t} \zeta^{n}, \nabla \zeta^{n}\right) \leq K_{0} h^{-1}\left\|\partial_{t} \zeta^{n}\right\|\left\|D^{\frac{1}{2}}\left(\mathbf{u}^{n}\right) \nabla \zeta^{n}\right\| .
$$


Hence by (3.72)-(3.75) we find that

$$
\begin{aligned}
\left\|\phi^{\frac{1}{2}} \partial_{t} \zeta^{n}\right\|^{2} & +\frac{1}{\Delta t}\left[\left\|D^{\frac{1}{2}}\left(\mathbf{u}^{n}\right) \nabla \zeta^{n}\right\|^{2}-\left\|D^{\frac{1}{2}}\left(\mathbf{u}^{n-1}\right) \nabla \zeta^{n-1}\right\|^{2}\right] \\
\leq \frac{K}{\bar{\epsilon}} & \left\{\left\|\mathbf{u}_{t}\right\|_{\infty} K_{0} h^{-2}\left\|\zeta^{n-1}\right\|^{2}+K_{0}^{2} h^{-2}\left(\left\|\zeta^{n-1}\right\|^{2}+\left\|\xi^{n-1}\right\|^{2}\right)+\left\|\zeta^{n}\right\|^{2}\right. \\
& \left.+\left\|\xi^{n}\right\|^{2}+\Delta t\left\|c_{\tau \tau}\right\|_{L^{2}\left(t^{n-1}, t^{n} ; L^{2}\right)}^{2}+(\Delta t)^{-1}\left\|\xi_{t}\right\|_{L^{2}\left(t^{n-1}, t^{n} ; L^{2}\right)}^{2}\right\} \\
& +\frac{K K_{0}^{2} h^{-2}}{\bar{\epsilon}}\left\|D^{\frac{1}{2}}\left(\mathbf{u}^{n}\right) \nabla \zeta^{n}\right\|^{2}-\Delta t\left\|D^{\frac{1}{2}}\left(\mathbf{u}^{n}\right) \nabla \partial_{t} \zeta^{n}\right\|^{2} \\
& +6 \bar{\epsilon}\left\|\partial_{t} \zeta^{n}\right\|^{2}
\end{aligned}
$$

where $\bar{\epsilon}>0$. We now choose $\bar{\epsilon}=\frac{\phi_{*}}{12}$ and hide the last term on the right-hand side of (3.76).

Multiplying the result by $\Delta t$ and summing on $n$, we derive the following

$$
\begin{aligned}
& \frac{\phi_{*}}{2}\left\|\partial_{t} \zeta\right\|_{L^{2}\left(L^{2}\right)}^{2}+\left\|D^{\frac{1}{2}}\left(\mathbf{u}^{N}\right) \nabla \zeta^{N}\right\|^{2} \\
& \leq K\left[h^{-2}\left|\|\zeta\|_{L^{2}\left(L^{2}\right)}^{2}+h^{-2}\|\mid \xi\|_{L^{2}\left(L^{2}\right)}^{2}+(\Delta t)^{2}\left\|c_{\tau \tau}\right\|_{L^{2}\left(L^{2}\right)}^{2}+\left\|\xi_{t}\right\|_{L^{2}\left(L^{2}\right)}^{2}\right]\right. \\
& \quad+K K_{0}^{2} h^{-2}\left\|D^{\frac{1}{2}}(\mathrm{u}) \nabla \zeta\right\|_{L^{2}\left(L^{2}\right)}^{2} .
\end{aligned}
$$

Thus, multiplying (3.77) by $h^{4}$, dividing by $\phi_{*} / 2$ and combining the result with (3.70), (3.7), and (3.8) we obtain

$$
\begin{aligned}
\left\|\partial_{t} \zeta\right\|_{L^{2}\left(L^{2}\right) \leq} & K h^{2}(\Delta t)^{2}\left\|c_{\tau \tau}\right\|_{L^{2}\left(L^{2}\right)}^{2}+K K_{0}^{2}\left(h^{2}+\epsilon\right)\|\| \phi^{\frac{1}{2}} e\|\|_{L^{2}\left(L^{2}\right)}^{2} \\
& +K h^{2(r+1)}\left(\left(h^{2}+\epsilon\right)\|c\|_{L^{2}\left(H^{r+1}\right)}^{2}+\|c\|_{H^{1}\left(H^{r-1}\right)}^{2}\right) .
\end{aligned}
$$

Combining (3.60), (3.62), (3.70), and (3.78), and noting the $\left\|e^{0}\right\|=\left\|\xi^{0}\right\|=$ $\mathcal{O}\left(h^{r+1}\right)$ by $(3.14),(3.6)$, and $(2.1)$, we find that

$$
\begin{gathered}
\left\|\mid \phi^{\frac{1}{2}} e\right\| \|_{L^{2}\left(L^{2}\right)}^{2} \leq K \\
K_{10}^{2}\left[\Delta t^{2}+h^{2(r+1)}\left(\||| c\|_{L^{2}\left(H^{(r+1)}\right)}^{2}+\|c\|_{H^{1}\left(H^{r-1}\right)}^{2}+\left\|c^{0}\right\|_{r+1}^{2}\right)\right] \\
+K K_{10}^{2} K_{0}^{2}\left(h^{2}+\epsilon\right)\left\|\phi^{\frac{1}{2}} e\right\|_{L^{2}\left(L^{2}\right)}^{2} .
\end{gathered}
$$

We now choose $\epsilon$ and $h$ so that $K K_{10}^{2} K_{0}^{2}\left(h^{2}+\epsilon\right)=\frac{1}{2}$.

Thus, we obtain the following result.

Theorem 3.3 Let the hypothesis of Lemma 3.2 hold. Assume

$$
c \in H^{2}\left(L^{2}\right) \cap L_{D}^{2}\left(H^{s+1}\right) \cap H^{1}\left(H^{s-1}\right)
$$

for some $s \geq 1$. Then for $h$ sufficiently small $\left.\left\||| \phi^{\frac{1}{2}} e\right\|\right|_{L^{2}\left(L^{2}\right)} \leq K_{11}\left[h^{r+1}\left(\||| c \mid\|_{L^{2}\left(H^{r+1}\right)}+\left\|c_{t}\right\|_{L^{2}\left(H^{r-1}\right)}+\left\|c^{0}\right\|_{r+1}\right)+\Delta t\left\|c_{\tau \tau}\right\|_{L^{2}\left(L^{2}\right)}\right]$, where $r=\min \left(k^{* *}, s\right)$, and

$$
K_{11}=K_{11}\left(K_{0}, K_{4}, K_{5}, K_{6}, K_{7}, K_{8}, K_{9}, K_{10}\right) .
$$




\section{Extensions}

Now assume in (1.1) that $\mu=\mu(c)$ is smooth, and we approximate $\mathbf{u}$ by a mixed finite element method. Let $\mathcal{V}^{h_{p}} \subset H(d i v ; \Omega)$ and $\mathcal{W}^{h_{p}} \subset L^{2}(\Omega)$ be mixed finite element spaces that approximate to order $h_{p}^{k+1}$ in $H(d i v)$ and $L^{2}$, respectively, with $\operatorname{div} \mathcal{V}^{h_{p}} \subset \mathcal{W}^{h_{p}}$. Thus, given a concentration approximation $C^{n}$ at time $t^{n}$, we find $\mathbf{U}^{n} \in \mathcal{V}^{h_{p}}$ and $P^{n} \in \mathcal{W}^{h_{p}}$ such that

$$
\left.\begin{array}{ll}
\left(\frac{\mu\left(C^{n}\right)}{k} \mathbf{U}^{n}, \mathbf{v}\right)-\left(P^{n}, \nabla \cdot \mathbf{v}\right)=0, & \mathbf{v} \in \mathcal{V}^{h_{p}}, \\
\left(\nabla \cdot \mathbf{U}^{n}, w\right)=\left(q^{n}, w\right), & w \in \mathcal{W}^{h_{p}} .
\end{array}\right\}
$$

Existence and uniqueness of $\mathrm{U}^{n}$ and $P^{n}$ is proved in [2]. By defining projections $\tilde{\mathbf{U}} \in \mathcal{V}^{h_{p}}, \tilde{P} \in \mathcal{W}^{h_{p}}$ via

$$
\left.\begin{array}{ll}
\left(\frac{\mu(c)}{k} \tilde{\mathbf{U}}, \mathbf{v}\right)-(\tilde{P}, \nabla \cdot \mathbf{v})=0, & \mathbf{v} \in \mathcal{V}^{h_{p}}, \\
(\nabla \cdot \tilde{\mathbf{U}}, w)=\left(q^{n}, w\right), & w \in \mathcal{W}^{h_{\mathrm{p}}},
\end{array}\right\}
$$

one can show [2]

$$
\begin{aligned}
& \|\mathbf{u}-\tilde{\mathbf{U}}\|_{L^{s}(H(d i v))}+\|p-\tilde{P}\|_{L^{\bullet}\left(L^{2}\right)} \\
& \quad \leq K h_{P}^{k+1}\left(\|\mathbf{u}\|_{L^{s}\left(H^{k+1}(d i v)\right)}+\|p\|_{L^{\cdot}\left(H^{k+1}\right)}\right)
\end{aligned}
$$

where $1 \leq s \leq \infty$. We remark that different mesh spacing can be used for $\mathcal{V}^{h_{p}}$ and $\mathcal{W}^{h_{p}}$ than for $\mathcal{M}_{h_{c}}$; i.e., we can have two different quasi-uniform meshes for pressurevelocity and concentration with diameters $\leq h_{p}$ and $h_{c}$, respectively.

Moreover, comparing (4.1) and (4.2) one obtains

$$
\left\|\mathbf{U}^{n}-\check{\mathbf{U}}^{n}\right\|_{H(d i v)}+\left\|P^{n}-\tilde{P}^{n}\right\| \leq K\left(1+\|\tilde{\mathbf{U}}\|_{\infty}\right)\left\|c^{n}-C^{n}\right\| .
$$

We remark that in the case $\mu(c) \equiv$ constant then $\mathbf{U}^{n}=\tilde{\mathbf{U}}^{n}$ and $P^{n}=\tilde{P}^{n}$.

We now solve the pressure-velocity and concentration equations in (1.1) sequentially. This sequential procedure is defined as follows. For simplicity, assume pressurevelocity and concentration are approximated using the same time-step $\Delta t$. Thus, given $C^{n-1}$, we calculate $\mathbf{U}^{n-1}$ and $P^{n-1}$ as in (4.1). Before calculating $C^{n}$ via the $M M O C$, we define an extrapolated velocity $E U^{n}$ by

$$
E \mathbf{U}^{n}= \begin{cases}2 \mathbf{U}^{n-1}-\mathbf{U}^{n-2}, & n>1, \\ \mathbf{U}^{0}, & n=1 .\end{cases}
$$

We remark that different time steps can be used for the calculation of $\mathbf{U}$ and $P$ than for $C$. In many practical problems the vector $\mathbf{U}$ may change less rapidly in time than $C$, even if the characteristics are taken into account. Thus, it is appropriate to use a larger time step for (4.1) than for (3.14). Partition $J$ into pressure time steps $0=t_{0}<t_{1}<\ldots<t_{M}=T$ with $\Delta t_{p}=t_{m}-t_{m-1}$. Each pressure step is assumed to also be a concentration step; i.e., for each $m$ there exists $n$ such that $t_{m}=t^{n}$. In 
general $\Delta t_{p}>\Delta t_{c}$. In this case we modify our definition of $E \mathbf{U}^{n}$ for $n>1$ and $m \geq 2$ as

$$
E \mathbf{U}^{n}=\left(1+\frac{t^{n}-t_{m-1}}{t_{m-1}-t_{m-2}}\right) \mathbf{U}_{m-1}-\frac{t^{n}-t_{m-1}}{t_{m-1}-t_{m-2}} \mathbf{U}_{m-2}
$$

Define

$$
\hat{\mathbf{x}}=\mathbf{x}-\frac{E \mathbf{U}^{n}(\mathbf{x})}{\phi(\mathbf{x})} \Delta t, \quad \overline{\mathbf{x}}=\mathbf{x}-\frac{E \mathbf{u}^{n}(\mathbf{x})}{\phi(\mathbf{x})} \Delta t
$$

and

$$
\hat{C}^{n-1}(\mathbf{x})=C^{n-1}(\hat{\mathbf{x}}), \quad \dot{C}^{n-1}(\mathbf{x})=C^{n-1}(\overline{\mathbf{x}}) .
$$

Then, $C^{n}, n \geq 1$ is given by

$$
\begin{array}{r}
\left(\phi \frac{C^{n}-\hat{C}^{n-1}}{\Delta t}, \chi\right)+\left(D\left(E \mathbf{U}^{n}\right) \nabla C^{n}, \nabla \chi\right)=\left(\tilde{q}^{n}\left(\tilde{c}^{n}-C^{n}\right), \chi\right), \\
\chi \in \mathcal{M}_{h}, \quad n \geq 1,
\end{array}
$$

\section{$4.1 \quad L_{D}^{\infty}\left(L^{2}\right)$ estimate}

In Theorem 3.1, we derived a suboptimal $L_{D}^{\infty}\left(L^{2}\right)$ estimate for the concentration error $e$ that allowed $D$ to go zero but assumed a given velocity $u$. We now consider the additional complications that arise when $\mathbf{u}$ is approximated by a mixed finite element solution $\mathbf{U}$. For simplicity, let $\Delta t_{p}=\Delta t_{\mathrm{c}}=\Delta t$. The theorem is as follows.

Theorem 4.1 Let the assumptions of Theorem 3.1 hold with the extra assumption $c \in L^{2}\left(W_{\infty}^{1}\right) \cap L^{\infty}\left(H^{1}\right)$. Let the numerical velocity $\mathbf{U}$ and the projection $\tilde{\mathbf{U}}$ be defined by (4.1) and (4.2) and assume $\mathrm{u} \in L^{2}\left(H^{k+1}(\right.$ div $\left.)\right), p \in L^{2}\left(H^{k+1}\right)$, and $\mathrm{u}_{t t} \in L^{2}\left(L^{2}\right)$.

If $\mu$ is independent of $c$ (as in the contaminant-transport problem), and $D$ is independent of $\mathrm{u}$ (i.e., $D$ can be zero or can consist of molecular diffusion only), then, with the additional assumption that

$$
h_{p}^{k+1}=\mathcal{O}\left(h_{c}^{2}\right)
$$

we obtain the estimate

$$
\|e\|_{L^{\infty}\left(L^{2}\right)} \leq K\left(h_{c}^{r}+h_{p}^{k+1}+\Delta t\right) .
$$

If $D$ depends on $\mathbf{u}$, the estimate takes the form

$$
\|\| e \|_{L^{\infty}\left(L^{2}\right)} \leq K\left(h_{c}^{r}+h_{c}^{-1} h_{p}^{k+1}+h_{c}^{-1} \Delta t^{2}+\Delta t\right) ;
$$

assuming (4.11) below, (4.9) still holds. 

and

If $\mu=\mu(c)$ and $D$ is independent of $\mathbf{u}$, then (4.9) holds provided that (4.8) holds

$$
r \geq 3, \quad h_{p}^{k+1}=o\left(h_{c}^{2}\right), \quad \Delta t=o\left(h_{c}^{2}\right),
$$

where $r=\min \left(s, k^{* *}\right)$.

If $\mu$ depends on $c$ and $D$ takes the following form that includes a nonzero hydrodynamic dispersion term,

$$
D(\mathbf{x}, \mathbf{u})=D_{m}(\mathbf{x})+\alpha_{l}|\mathbf{u}|^{\beta}\left[\begin{array}{cc}
\hat{u}_{x}^{2} & \hat{u}_{x} \hat{u}_{y} \\
\hat{u}_{x} \hat{u}_{y} & \hat{u}_{y}^{2}
\end{array}\right]+\alpha_{t}|\mathbf{u}|^{\beta}\left[\begin{array}{cc}
\hat{u}_{y}^{2} & -\hat{u}_{x} \hat{u}_{y} \\
-\hat{u}_{x} \hat{u}_{y} & \hat{u}_{x}^{2}
\end{array}\right],
$$

where no lower bound is assumed for $D_{m}$ and $\hat{u}_{x}$ and $\hat{u}_{y}$ represent the direction cosine and sine of $\mathrm{u}$, then (4.9) holds provided that (4.8) and (4.11) are satisfied and

$$
\alpha_{l}^{2} / \alpha_{t} \leq \alpha^{*}<\infty, \quad \alpha_{t}^{2} / \alpha_{l} \leq \alpha^{*}<\infty, \quad \beta \geq 2
$$

Proof. We add the necessary details to the proof of Theorem 3.1. Some manipulations lead to an analogue of $(3.26)$, where $D\left(E \mathbf{U}^{n}\right)$ replaces $D\left(\mathbf{u}^{n}\right)$ on the left-hand side and the right-hand side has the additional terms

$$
\begin{gathered}
\left(\left[\mathbf{u}^{n}-E \mathbf{u}^{n}\right] \cdot \nabla c^{n}, \zeta^{n}\right)+\left(\left[D\left(\mathbf{u}^{n}\right)-D\left(E \mathbf{U}^{n}\right)\right] \nabla \tilde{C}^{n}, \nabla \zeta^{n}\right) \\
-\left(\phi \frac{\check{\tilde{C}}^{n-1}-\hat{\tilde{C}}^{n-1}}{\Delta t}, \zeta^{n}\right)-\left(\phi \frac{\check{\zeta}^{n-1}-\hat{\zeta}^{n-1}}{\Delta t}, \zeta^{n}\right) \\
\equiv T_{2}+T_{3}+\left(T_{7}+T_{8}\right)+T_{9}
\end{gathered}
$$

the terms are numbered in accordance with the corresponding terms in [5]. The result will follow as in Theorem 3.1 once we estimate the new terms.

As in [5], we have

$$
\begin{aligned}
\left|T_{2}\right| & \leq\left\|\mathrm{u}^{n}-E \mathrm{u}^{n}\right\|\left\|\nabla c^{n}\right\|_{\infty}\left\|\zeta^{n}\right\| \\
& \leq K \Delta t^{3}\left\|u_{t t}\right\|_{L^{2}\left(t^{n-2}, t^{n} ; L^{2}\right)}^{2}\left\|\nabla c^{n}\right\|_{\infty}^{2}+K\left\|\zeta^{n}\right\|^{2} .
\end{aligned}
$$

Arguments like those in [5] also show that

$$
\begin{aligned}
\left|T_{7}+T_{8}\right| & \leq K\left\|\nabla \tilde{C}^{n-1}\right\|_{\infty}\left\|E\left(\mathbf{u}^{n}-\mathbf{U}^{n}\right)\right\|\left\|\zeta^{n}\right\| \\
& \leq K\left\|E\left(\mathbf{u}^{n}-\mathbf{U}^{n}\right)\right\|^{2}+K\left\|\zeta^{n}\right\|^{2} \\
& \leq K h_{p}^{2 k+2}+K\left\|E \tilde{U}^{n}-E \mathbf{U}^{n}\right\|^{2}+K\left\|\zeta^{n}\right\|^{2}
\end{aligned}
$$

For $T_{3}$, note first that $T_{3}=0$ if $D$ is independent of $\mathbf{u}$. Otherwise, we write

$$
T_{3}=\int_{\Omega}\left[\int_{0}^{1} \frac{\partial D}{\partial \mathbf{u}}\left(\theta \mathbf{u}^{n}+(1-\theta) E \mathbf{U}^{n}\right) d \theta\right]\left(\mathbf{u}^{n}-E \mathbf{U}^{n}\right) \nabla \tilde{C}^{n} \nabla \zeta^{n} d \mathbf{x} .
$$


The factor $\mathbf{u}^{n}-E \mathbf{U}^{n}$ can be written as

$$
\mathbf{u}^{n}-E \mathbf{U}^{n}=\left(\mathbf{u}^{n}-E \mathbf{u}^{n}\right)+\left(E \mathbf{u}^{n}-E \tilde{\mathbf{U}}^{n}\right)+\left(E \tilde{\mathbf{U}}^{n}-E \mathbf{U}^{n}\right) ;
$$

we note that the last term is zero if $\mu$ is independent of $c$. Using bounds on $\partial D / \partial \mathbf{u}$ and $\nabla \tilde{C}^{n}$ with an inverse inequality for $\nabla \zeta^{n}$, we obtain

$$
\left|T_{3}\right| \leq K h_{c}^{-2}\left(\Delta t^{3}\left\|u_{t t}\right\|_{L^{2}\left(t^{n-2}, t^{n} ; L^{2}\right)}^{2}+h_{p}^{2 k+2}\right)+K\left\|\zeta^{n}\right\|^{2}
$$

when $\mu$ is independent of $c$.

If $\mu$ depends on $c$, we write $T_{3}$ in the form

$$
\begin{aligned}
T_{3}= & \int_{\Omega} \int_{0}^{1}\left[\frac{\partial D}{\partial \mathbf{u}}\left(\theta \mathbf{u}^{n}+(1-\theta) E \mathbf{U}^{n}\right)-\frac{\partial D}{\partial \mathbf{u}}\left(E \mathbf{U}^{n}\right)\right] d \theta\left(\mathbf{u}^{n}-E \mathbf{U}^{n}\right) \nabla \tilde{C}^{n} \nabla \zeta^{n} d \mathbf{x} \\
& \quad+\int_{\Omega} \frac{\partial D}{\partial \mathbf{u}}\left(E \mathbf{U}^{n}\right)\left(\mathbf{u}^{n}-E \mathbf{U}^{n}\right) \nabla \tilde{C}^{n} \nabla \zeta^{n} d \mathbf{x} \\
\equiv & W_{1}+W_{2} .
\end{aligned}
$$

Assuming $\partial^{2} D / \partial \mathbf{u}^{2}$ is bounded, we have (using inverse inequalities)

$$
\begin{aligned}
\left|W_{1}\right| & \leq K\left(\frac{\partial^{2} D}{\partial \mathbf{u}^{2}}\right)\left\|\mathbf{u}^{n}-E \mathbf{U}^{n}\right\|^{2}\left\|\nabla \zeta^{n}\right\|_{\infty} \\
& \leq K h_{c}^{-2}\left\|\mathbf{u}^{n}-E \mathbf{U}^{n}\right\|^{2}\left\|\zeta^{n}\right\| .
\end{aligned}
$$

With $r \geq 3$, since we ultimately show that $\left\|\zeta^{n}\right\|=\mathcal{O}\left(h_{c}^{r}+h_{p}^{k+1}+\Delta t\right)$, (4.11) implies that we have $h_{c}^{-2}\left\|\zeta^{n}\right\|=o(1)$ in (4.21); thus $W_{1}$ falls under the estimates for $T_{2}$ and $T_{7}+T_{8}$ above.

For $W_{2}$, we consider the strategy

$$
\begin{aligned}
\left|W_{2}\right| \leq \frac{1}{2} \int_{\Omega} & D\left(E \mathbf{U}^{n}\right) \nabla \zeta^{n} \nabla \zeta^{n} d \mathbf{x} \\
& +\frac{1}{2} \int_{\Omega} D\left(E \mathbf{U}^{n}\right)^{-1}\left|\frac{\partial D}{\partial \mathbf{u}}\left(E \mathbf{U}^{n}\right)\right|^{2}\left|\mathbf{u}^{n}-E \mathbf{U}^{n}\right|^{2}\left|\nabla \tilde{C}^{n}\right|^{2} d \mathbf{x} .
\end{aligned}
$$

With (4.13), we show that $D^{-1}\left|D_{\mathbf{u}}\right|^{2}$ is bounded, so that the first term in (4.22) hides on the left-hand side of the analogue of (3.26) and the second term leads to $K\left\|\mathbf{u}^{n}-E \mathbf{U}^{n}\right\|^{2}$; thus $W_{2}$, like $W_{1}$, is covered by the $T_{2}$ and $T_{7}+T_{8}$ estimates.

The one-dimensional analogue of $(4.12)$ is $D(x, u)=D_{m}(x)+\alpha(x)|u|^{\beta}$, so that

$$
\left|D^{-1}\left(\frac{\partial D}{\partial u}\right)^{2}\right| \leq \alpha^{-1}|u|^{-\beta}\left(\beta \alpha|u|^{\beta-1}\right)^{2}=\beta^{2} \alpha|u|^{\beta-2},
$$

which is bounded for $\beta \geq 2$. We seek a bound similar to (4.23) in two dimensions. To see the form of $\left(D_{\mathbf{u}}\right)^{2}$, assume for simplicity that $\mathbf{u}$ is oriented in the $x$-direction (rotation of coordinates will not affect the size of $\left.\left(D_{\mathbf{u}}\right)^{2}\right)$. Then

$$
D=D_{m}+|\mathbf{u}|^{\beta}\left[\begin{array}{cc}
\alpha_{l} & 0 \\
0 & \alpha_{t}
\end{array}\right], \quad D^{-1} \leq|\mathbf{u}|^{-\beta}\left[\begin{array}{cc}
\alpha_{l}^{-1} & 0 \\
0 & \alpha_{t}^{-1}
\end{array}\right],
$$




$$
\begin{gathered}
\frac{\partial D}{\partial u_{x}}=\beta|\mathbf{u}|^{\beta-1}\left[\begin{array}{cc}
\alpha_{l} & 0 \\
0 & \alpha_{t}
\end{array}\right], \quad \frac{\partial D}{\partial u_{y}}=|\mathbf{u}|^{\beta-1}\left[\begin{array}{cc}
0 & \alpha_{l}-\alpha_{t} \\
\alpha_{l}-\alpha_{t} & 0
\end{array}\right], \\
\left|D^{-1}\left(\frac{\partial D}{\partial \mathbf{u}}\right)^{2}\right| \leq|\mathbf{u}|^{\beta-2}\left(\beta^{2}\left[\begin{array}{cc}
\alpha_{l} & 0 \\
0 & \alpha_{t}
\end{array}\right]+\left[\begin{array}{cc}
\alpha_{l}^{-1}\left(\alpha_{l}-\alpha_{t}\right)^{2} & 0 \\
0 & \alpha_{t}^{-1}\left(\alpha_{l}-\alpha_{t}\right)^{2}
\end{array}\right]\right) .
\end{gathered}
$$

The desired bound follows as long as (4.13) holds.

Finally, $T_{9}$ leads to (via inverse inequalities)

$$
\begin{aligned}
\left|T_{9}\right| & \leq K\left\|\nabla \zeta^{n-1}\right\|\left\|E\left(\mathbf{u}^{n}-\mathrm{U}^{n}\right)\right\|\left\|\zeta^{n}\right\|_{\infty} \\
& \leq K h_{c}^{-2}\left\|E\left(\mathbf{u}^{n}-\mathrm{U}^{n}\right)\right\|\left\|\zeta^{n-1}\right\|\left\|\zeta^{n}\right\| \\
& \leq K h_{c}^{-2}\left(h_{p}^{k+1}+\left\|E \tilde{U}^{n}-E \mathrm{U}^{n}\right\|\right)\left\|\zeta^{n-1}\right\|\left\|\zeta^{n}\right\| .
\end{aligned}
$$

If $\mu$ is independent of $c$, the $\tilde{U}-U$ term does not appear in (4.24), so that (4.8) implies

$$
\left|T_{9}\right| \leq K\left(\left\|\zeta^{n-1}\right\|^{2}+\left\|\zeta^{n}\right\|^{2}\right)
$$

Note that the constant in (4.25) depends on pressure-related quantities only, which are independent of $c$, so that $\mathcal{O}\left(h_{c}^{2}\right)$ suffices in (4.8) and $o\left(h_{c}^{2}\right)$ is not necessary. On the other hand, if $\mu$ depends on $c$, we need the estimate

$$
\begin{aligned}
\left\|E \tilde{\mathbf{U}}^{n}-E \mathbf{U}^{n}\right\| & \leq K\left(\left\|\xi^{n-1}\right\|+\left\|\xi^{n-2}\right\|+\left\|\zeta^{n-1}\right\|+\left\|\zeta^{n-2}\right\|\right) \\
& \leq K\left(h_{c}^{r}+\left\|\zeta^{n-1}\right\|+\left\|\zeta^{n-2}\right\|\right) .
\end{aligned}
$$

Using (4.11) as in the $W_{1}$ estimate, we substitute (4.26) into (4.24) to obtain (4.25). Now, however, the constant contains $h_{c}^{-2}\|\zeta\|$; the $\zeta$-dependence requires that this be $o(1)$ in order to avoid unbounded growth when Gronwall's Lemma is applied. We also use $(4.26)$ in $(4.16)$ to obtain

$$
\left|T_{7}+T_{8}\right| \leq K\left(h_{p}^{2 k+2}+h_{c}^{2 r}+\sum_{i=0}^{2} \| \zeta^{n-i}||^{2}\right) .
$$

Combining (4.14), (4.15), (4.19), (4.25), and (4.27) in the appropriate cases, and recalling that $W_{1}$ and $W_{2}$ are covered by $T_{2}$ and $T_{7}+T_{8}$, we derive the theorem.

Remarks. The assumptions on $\alpha_{l}$ and $\alpha_{t}$ are in accord with physical reality (usually $\alpha_{l} \approx 10 \alpha_{t}$ ), but the assumption on $\beta$ is not. Typical values of $\beta$ are in the range of 1 to 1.2 [11] ((1.2) corresponds to $\beta=1$, which would not allow the $D^{-1}$ integral to be bounded. However, the trouble appears only where $u$ is small, in which case little is happening physically. It would seem that practical computations could modify $\alpha$ and $\beta$ for small $\mathbf{u}$ such that $D$ still depends smoothly on $\mathbf{u}$ but $\beta \geq 2$ for small $\mathbf{u}$; this would satisfy the conditions of the theorem without significantly changing the physics. 


\section{$4.2 \quad L_{D}^{2}\left(L^{2}\right)$ estimate}

Under the assumption $\mu$ is independent of $c$, we derive an estimate for $\|\mid\|_{L^{2}\left(L^{2}\right)}$, where $C^{n}$ is defined by (4.7) and $\mathbf{U}^{n}$ by (4.1). Recall from (4.5)

$$
\check{\mathbf{x}} \equiv G\left(\mathbf{x}, t^{n}\right)=\mathbf{x}-\frac{E \mathbf{u}^{n}(\mathbf{x})}{\phi(\mathbf{x})} \Delta t
$$

and $Z^{n}(\mathbf{x})$ again denotes $G^{-1}\left(\mathbf{x}, t^{n}\right)$. We again let $\psi^{n}, n=0,1, \ldots, N^{*}+1$, be defined as in (3.53). The bound (3.35) still holds and by emulating the arguments of Section 3, i.e. (3.53)-(3.59) with $\mathcal{M}_{h}=\mathcal{M}_{h_{c}}$ and $\psi_{I}$ an $H^{1}$ projection of $\psi$, we find for $e^{n}=c^{n}-C^{n}$,

$$
\begin{aligned}
& \left\|\phi^{\frac{1}{2}} e\right\|_{L^{2}\left(L^{2}\right)}^{2}=\sum_{n=1}^{N^{*}}\left\{\left(\phi \frac{e^{n}-\check{e}^{n-1}}{\Delta t}, \psi^{n}\right)+\left(D\left(\mathbf{u}^{n}\right) \nabla e^{n}, \nabla \psi^{n}\right)+\left(\tilde{q}^{n} \psi^{n}, e^{n}\right)\right\} \Delta t \\
& =\sum_{n=1}^{N^{*}}\left\{\left(\phi \frac{e^{n}-\dot{e}^{n-1}}{\Delta t}, \psi^{n}-\psi_{I}^{n}\right)+\left(D\left(\mathbf{u}^{n}\right) \nabla e^{n}, \nabla\left(\psi^{n}-\psi_{I}^{n}\right)\right)\right. \\
& +\left(\tilde{q}^{n} e^{n}, \psi^{n}-\psi_{I}^{n}\right)+\left(\phi \ddot{e}^{0}, \psi^{1}\right) \\
& \left.+\left(\tilde{\sigma}^{n}, \psi_{I}^{n}\right)-\left(\rho^{n}, \nabla \psi_{I}^{n}\right)\right\} \Delta t,
\end{aligned}
$$

where

$$
\begin{aligned}
\tilde{\sigma}^{n} & =\left[-\phi \frac{c^{n}-\check{c}^{n-1}}{\Delta t}+\left(\phi c_{t}^{n}+E \mathbf{u}^{n} \cdot \nabla c^{n}\right)\right]+\left(\mathbf{u}^{n}-E \mathbf{u}^{n}\right) \cdot \nabla c^{n}+\phi \frac{\check{C}^{n-1}-\hat{C}^{n-1}}{\Delta t} \\
& \equiv \sigma_{1}^{n}+\sigma_{2}^{n}+\sigma_{3}^{n},
\end{aligned}
$$

and

$$
\rho^{n}=\left(D\left(\mathbf{u}^{n}\right)-D\left(E \mathbf{U}^{n}\right)\right) \nabla C^{n} .
$$

Now

$$
\begin{aligned}
\left(\tilde{\sigma}^{n}, \psi_{I}^{n}\right) & \\
= & \left(\sigma_{1}^{n}, \psi_{I}^{n}\right)+\left(\sigma_{2}^{n}, \psi_{I}^{n}\right)+\left(\phi \frac{\check{\zeta}^{n-1}-\hat{\zeta}^{n-1}}{\Delta t}, \psi_{I}^{n}\right)-\left(\phi \frac{\check{\xi}^{n-1}-\hat{\xi}^{n-1}}{\Delta t}, \psi_{I}^{n}\right) \\
& +\left(\phi \frac{\check{c}^{n-1}-\hat{c}^{n-1}}{\Delta t}, \psi_{I}^{n}\right) .
\end{aligned}
$$

As before

$$
\left|\left(\sigma_{1}^{n}, \psi_{I}^{n}\right)\right| \leq K \Delta t|| c_{\tau \tau}\left\|_{L^{2}\left(t^{n-1}, t^{n} ; L^{2}\right)}^{2}+\delta\right\| \psi^{n} \|_{1}^{2} .
$$

Moreover, we have

$$
\left|\left(\sigma_{2}^{n}, \psi_{I}^{n}\right)\right| \leq K\left(\Delta t_{p}\right)^{3 / 2}\left\|u_{t t}\right\|_{L^{2}\left(t_{m-2}, t_{m} ; L^{2}\right)}\left\|\nabla c^{n}\right\|_{\infty}\left\|\psi^{n}\right\|_{1},
$$


thus

$$
\sum_{n=1}^{N^{*}}\left|\left(\sigma_{2}^{n}, \psi_{I}^{n}\right)\right| \Delta t \leq\left. K \Delta t_{p}^{3} \Delta t|| u_{t t}\right|_{L^{2}\left(L^{2}\right)} ^{2}|||c|\left\|_{L^{2}\left(W_{\infty}^{1}\right)}^{2}+\delta \mid\right\| \psi\|\|_{L^{\infty}\left(H^{1}\right)}^{2} .
$$

Following arguments given in [5], we see that the last three terms in (4.31) are bounded by

$$
\left\|E\left(\mathbf{u}^{n}-\mathbf{U}^{n}\right)\right\|\left(\left\|g_{c}\right\|_{\infty}\left\|\psi_{I}^{n}\right\|+\left\|g_{\varepsilon}\right\|\left\|\psi_{I}^{n}\right\|_{\infty}+\left\|g_{\zeta}\right\|\left\|\psi_{I}^{n}\right\|_{\infty}\right)
$$

where

$$
g_{\theta}(\mathbf{x})=\int_{0}^{1} \frac{\partial \theta^{n-1}}{\partial \mathbf{z}}((1-\bar{z}) \check{\mathbf{x}}+\bar{z} \hat{\mathbf{x}}) d \bar{z},
$$

with $\mathbf{z}$ the unit vector in the direction of $\overline{\mathbf{x}}-\hat{\mathbf{x}}$. It was shown that

$$
\left\|g_{\theta}\right\| \leq K\left\|\nabla \theta^{n-1}\right\|
$$

ind

$$
\left\|g_{\theta}\right\|_{\infty} \leq K\left\|\nabla \theta^{n-1}\right\|_{\infty} .
$$

It is also well known (see Bramble [1]) that for $\chi \in \mathcal{M}_{h_{c}}$,

$$
\|\chi\|_{\infty} \leq K\left|\log h_{c}\right|^{\frac{1}{2}}\|\chi\|_{1} .
$$

Thus we see that (4.34) is bounded by

$$
K\left\|E\left(\mathbf{u}^{n}-\mathrm{U}^{n}\right)\right\|\left(\left\|\nabla c^{n-1}\right\|_{\infty}+\left|\log h_{c}\right|^{\frac{1}{2}}\left(\left\|\nabla \xi^{n-1}\right\|+\| \nabla \zeta^{n-1}||\right)\right)\left\|\psi^{n}\right\|_{1} ;
$$

hence

$$
\begin{aligned}
& \sum_{n=1}^{N^{*}}\left|\sigma_{3}^{n}\right| \Delta t \\
& \leq K \mid\|E(\mathbf{u}-\mathbf{U})\| \|_{L^{2}\left(L^{2}\right)}^{2}\left[\|\| c\left|\|_{L^{2}\left(W_{\infty}^{1}\right)}^{2}+\right| \log h_{c} \mid\left(\|\| \nabla \zeta\left\|_{L^{2}\left(L^{2}\right)}^{2}+\right\|\|\nabla \xi\|_{L^{2}\left(L^{2}\right)}^{2}\right)\right] \\
& +\delta \mid\|\psi\| \|_{L^{\infty}\left(H^{1}\right)}^{2}
\end{aligned}
$$

Combining (4.31), (4.32), (4.33), and (4.36), we find that

$$
\begin{aligned}
& \sum_{n=1}^{N^{*}}\left|\left(\tilde{\sigma}^{n}, \psi_{I}^{n}\right)\right| \Delta t \\
& \leq K\left\{\Delta t^{2}\left\|c_{\tau \tau}\right\|_{L^{2}\left(L^{2}\right)}^{2}+\Delta t_{p}^{3} \Delta t\left\|u_{t t}\right\|_{L^{2}\left(L^{2}\right)}^{2}\|c \mid\|_{L^{2}\left(W_{\infty}^{1}\right)}^{2}\right. \\
&+\|E(\mathbf{u}-\mathbf{U})\|_{L^{2}\left(L^{2}\right)}^{2}\left[\left\|c\left|\|_{L^{2}\left(W_{\infty}^{1}\right)}^{2}+\right| \log h_{c} \mid\left(\|\nabla \zeta\|_{L^{2}\left(L^{2}\right)}^{2}+\|\| \nabla \xi \|_{L^{2}\left(L^{2}\right)}^{2}\right)\right]\right\} \\
&+3 \delta\|\psi\| \|_{L^{\infty}\left(H^{1}\right)}^{2} .
\end{aligned}
$$


We have

$$
\begin{aligned}
& \left|\left(\rho^{n}, \nabla \psi_{I}^{n}\right)\right| \\
& \leq\left|\left(\left(D\left(\mathbf{u}^{n}\right)-D\left(E \mathbf{U}^{n}\right)\right) \nabla c^{n}, \nabla \psi_{I}^{n}\right)\right|+\left|\left(\left(D\left(\mathbf{u}^{n}\right)-D\left(E \mathbf{U}^{n}\right)\right) \nabla e^{n}, \nabla \psi_{I}^{n}\right)\right| \\
& \leq K\left\|D_{\mathbf{u}}\right\|_{\infty}\left\|\nabla \psi^{n}\right\|\left\|\mathbf{u}^{n}-E \mathbf{U}^{n}\right\| \\
& \quad \times\left(\left\|\nabla c^{n}\right\|_{\infty}+\left|\log h_{c}\right|^{\frac{1}{2}}\left(\left\|\nabla \xi^{n}\right\|+\left\|\nabla \zeta^{n}\right\|\right)\right) .
\end{aligned}
$$

Now

$$
\begin{aligned}
\left\|\mathbf{u}^{n}-E \mathbf{U}^{n}\right\| & \leq\left\|\mathbf{u}^{n}-E \mathbf{u}^{n}\right\|+\left\|E\left(\mathbf{u}^{n}-\mathbf{U}^{n}\right)\right\| \\
& \leq K \Delta t_{p}^{3 / 2}\left\|u_{t t}\right\|_{L^{2}\left(t_{m-2}, t_{m} ; L^{2}\right)}+\left\|E\left(\mathbf{u}^{n}-\mathbf{U}^{n}\right)\right\| .
\end{aligned}
$$

Combining (4.38) and (4.39) we see that

$$
\begin{aligned}
& \sum_{n=1}^{N^{*}}\left|\left(\rho^{n}, \nabla \psi_{I}^{n}\right)\right| \Delta t \\
& \leq K\left[\|\| E(\mathbf{u}-\mathbf{U})\left\|_{L^{2}\left(L^{2}\right)}^{2}+\Delta t \Delta t_{p}^{3}\right\| u_{t i} \|_{L^{2}\left(L^{2}\right)}^{2}\right] \\
& \times\left(\|\| c\left|\|_{L^{2}\left(W_{\infty}^{1}\right)}^{2}+\right| \log h_{c} \mid\left(\|\| \nabla \xi\left\|_{L^{2}\left(L^{2}\right)}^{2}+\right\|\|\nabla \zeta\|_{L^{2}\left(L^{2}\right)}^{2}\right)\right) \\
& \quad+\delta\|\| \psi \|_{L^{\infty}\left(H^{1}\right)}^{2},
\end{aligned}
$$

where $K=K\left(\left\|D_{\mathbf{u}}\right\|_{\infty}\right)$.

From (4.28), (4.37), and (4.40), and Lemma 3.2 we deduce that

$$
\begin{aligned}
& \|e\|_{L^{2}\left(L^{2}\right)}^{2} \\
& \leq K\left\{\Delta t^{2}\left\|c_{\tau \tau}\right\|_{L^{2}\left(L^{2}\right)}^{2}+h^{4}\left\|\partial_{t} e\right\|\left\|_{L^{2}\left(L^{2}\right)}^{2}+h^{2}\right\|\|e\|_{L^{\infty}\left(L^{2}\right)}^{2}\right. \\
& \left.+h^{2}\|\| e\left\|_{L^{2}\left(H^{1}\right)}^{2}+\right\| e^{0} \|^{2}\right\} \\
& +K\left[\|\| E\left(\mathbf{u}^{n}-\mathbf{U}^{n}\right)\left\|_{L^{2}\left(L^{2}\right)}^{2}+\Delta t \Delta t_{p}^{3}\right\| u_{t t} \|_{L^{2}\left(L^{2}\right)}^{2}\right] \\
& \times\left(\|\| c \|_{L^{2}\left(W_{\infty}^{1}\right)}^{2}+\left|\log h_{c}\right|\left(\||| \nabla \xi\|_{L^{2}\left(L^{2}\right)}^{2}+\|\| \nabla \zeta \|_{L^{2}\left(L^{2}\right)}^{2}\right)\right) .
\end{aligned}
$$

We now estimate $h_{c}\left\|\left|\|\|_{L^{2}\left(H^{1}\right)}, h_{c}\|\mid e\|_{L^{\infty}\left(L^{2}\right)}\right.\right.$, and $h_{c}^{2}\|\| \partial_{t} e \|_{L^{2}\left(L^{2}\right)}$. We have

$$
\begin{aligned}
\left(\phi \partial_{t} \zeta^{n}, \chi\right)+\left(D\left(E \mathbf{U}^{n}\right) \nabla \zeta^{n}, \nabla \chi\right)+\left(\tilde{q} \zeta^{n}, \chi\right) & \\
= & -\left(\sigma_{1}^{n}, \chi\right)+\left(\left[\mathbf{u}^{n}-E \mathbf{u}^{n}\right] \cdot \nabla c^{n}, \chi\right)+\left(\left[D\left(\mathbf{u}^{n}\right)-D\left(E \mathbf{U}^{n}\right)\right] \nabla \tilde{C}^{n}, \nabla \chi\right) \\
& \quad+\left(\phi \partial_{t} \xi^{n}, \chi\right)-\left(\xi^{n}, \chi\right) \\
- & \left(\phi \frac{\check{c}^{n-1}-\hat{c}^{n-1}}{\Delta t}, \chi\right)+\left(\phi \frac{\check{\xi}^{n-1}-\hat{\xi}^{n-1}}{\Delta t}, \chi\right)-\left(\phi \frac{\check{\zeta}^{n-1}-\hat{\zeta}^{n-1}}{\Delta t}, \chi\right) \\
& -\left(\phi \frac{\check{\xi}^{n-1}-\xi^{n-1}}{\Delta t}, \chi\right)+\left(\phi \frac{\check{\zeta}^{n-1}-\zeta^{n-1}}{\Delta t}, \chi\right), \quad \chi \in \mathcal{M}_{h_{c}} .
\end{aligned}
$$

where $\sigma_{1}^{n}$ is given by $(4.29)$. 
There are five terms in (4.42) not present in (3.63); namely

$$
\begin{gathered}
\left(\phi \frac{\check{g}^{n-1}-\hat{g}^{n-1}}{\Delta t}, \chi\right), \quad g=c, \xi, \zeta, \\
\left(\left[\mathbf{u}^{n}-E \mathbf{u}^{n}\right] \cdot \nabla c^{n}, \chi\right),
\end{gathered}
$$

and

$$
\left(\left[D\left(\mathbf{u}^{n}\right)-D\left(E \mathbf{U}^{n}\right)\right] \nabla \tilde{C}^{n}, \nabla \chi\right) .
$$

To estimate $h_{c}\|e \mid\|_{L^{\infty}\left(L^{2}\right)}$, and $h_{c}\|e \mid\|_{L^{2}\left(H^{1}\right)}$ in (4.41) we set $\chi=\zeta^{n}$ in (4.42). In addition, to estimate $h_{c}^{2}\left\|\mid \partial_{t} e\right\|_{L^{2}\left(L^{2}\right)}$ we set $\chi=\partial_{t} \zeta^{n}$ in (4.42) and then use the triangle inequality.

As in the derivation of the bound for (4.31), we see that

$$
\begin{aligned}
T_{5}^{n} \equiv & \left|\left(\phi \frac{\check{c}^{n-1}-\hat{c}^{n-1}}{\Delta t}, \zeta^{n}\right)\right|+\left|\left(\phi \frac{\dot{\xi}^{n-1}-\hat{\xi}^{n-1}}{\Delta t}, \zeta^{n}\right)\right| \\
& \quad+\left|\left(\phi \frac{\zeta^{n-1}-\hat{\zeta}^{n-1}}{\Delta t}, \zeta^{n}\right)\right| \\
\leq & \left\|E\left(\mathbf{u}^{n}-\mathbf{U}^{n}\right)\right\|\left(\left\|\nabla c^{n-1}\right\|+\left\|\nabla \xi^{n-1}\right\|+\left\|\nabla \zeta^{n-1}\right\|\right)\left\|\zeta^{n}\right\|_{\infty} .
\end{aligned}
$$

Applying inverse assumptions we have

$$
\begin{gathered}
T_{5}^{n} \leq K\left\|E\left(\mathbf{u}^{n}-\mathrm{U}^{n}\right)\right\|^{2}\left(\left\|\nabla \xi^{n-1}\right\|^{2}+\left\|\nabla \zeta^{n-1}\right\|^{2}+\left\|\nabla c^{n-1}\right\|^{2}\right) \\
+\epsilon h_{c}^{-2}\left\|\zeta^{n}\right\|^{2} .
\end{gathered}
$$

Thus by approximation theory on $\xi$, inverse assumptions on $\zeta$, and Schwarz's inequality

$$
h_{c}^{2} \sum_{n=1}^{N} T_{5}^{n} \Delta t \leq K\left(h_{c}^{2}\|\| c\left\|_{L^{4}\left(H^{1}\right)}^{2}+\right\| \zeta\|\|_{L^{4}\left(L^{2}\right)}^{2}\right)\|\| \mathbf{u}-\mathbf{U}\left\|_{L^{4}\left(L^{2}\right)}^{2}+\epsilon\right\| \zeta \|_{L^{2}\left(L^{2}\right)}^{2} .
$$

Now

$$
T_{6}^{n} \equiv\left|\left(\left[\mathbf{u}^{n}-E \mathbf{u}^{n}\right] \cdot \nabla c^{n}, \zeta^{n}\right)\right| \leq \Delta t_{p}^{3 / 2}\left\|u_{t t}\right\|_{L^{2}\left(t_{m-2}, t_{m} ; L^{2}\right)}\left\|\nabla c^{n}\right\|_{\infty}\left\|\zeta^{n}\right\|
$$

and

$$
h_{c}^{2} \sum_{n=1}^{N^{*}} T_{6}^{n} \Delta t \leq K h_{c}^{2} \Delta t_{p}^{3}\left\|u_{t t}\right\|_{L^{2}\left(L^{2}\right)}^{2}\|c \mid\|_{L^{2}\left(W_{\infty}^{1}\right)}^{2}+K h_{c}^{2}\|\| \zeta \|_{L^{2}\left(L^{2}\right)}^{2} .
$$

Similarly

$$
\left|\left(\left[D\left(\mathbf{u}^{n}\right)-D\left(E \mathbf{U}^{n}\right)\right] \nabla \tilde{C}^{n}, \nabla \zeta^{n}\right)\right| \leq\left\|D_{\mathbf{u}}^{n}\right\|_{\infty}\left\|\mathbf{u}^{n}-E \mathbf{U}^{n}\right\|\left\|\nabla \tilde{C}^{n}\right\|_{\infty}\left\|\zeta^{n}\right\|_{1}
$$


and by inverse assumptions and approximation theory, we see that

$$
\begin{aligned}
& h_{c}^{2} \sum_{n=1}^{N^{*}}\left|\left(\left(D\left(\mathbf{u}^{n}\right)-D\left(E \mathbf{U}^{n}\right)\right) \nabla \tilde{C}^{n}, \nabla \zeta^{n}\right)\right| \Delta t \\
& \leq K \sum_{n=1}^{N^{*}}\left\|D_{\mathbf{u}}^{n}\right\|_{\infty}\left(\left\|\mathbf{u}^{n}-E \mathbf{u}^{n}\right\|+\left\|E \mathbf{u}^{n}-E \mathbf{U}^{n}\right\|\right)\left\|\nabla c^{n}\right\|\left\|\zeta^{n}\right\| \Delta t \\
& \leq K\left\|D_{\mathbf{u}}\right\| \|_{L^{\infty}\left(L^{\infty}\right)}\left(\Delta t_{p}^{3}\left\|u_{t t}\right\|_{L^{2}\left(L^{2}\right)}^{2}\|c\|_{L^{2}\left(H^{1}\right)}^{2}+\|\mathbf{u}-\mathbf{U}\|\left\|_{L^{4}\left(L^{2}\right)}^{2}\right\| c\|\|_{L^{4}\left(H^{1}\right)}^{2}\right) \\
& \quad+\epsilon\|\zeta\| \|_{L^{2}\left(L^{2}\right)} .
\end{aligned}
$$

Combining (4.42)-(4.45) and (3.69) we obtain, for arbitrary $N \leq N^{*}$,

$$
\begin{aligned}
& h_{c}^{2}\left\|\phi^{\frac{1}{2}} \zeta^{N}\right\|^{2}+h_{c}^{2} \sum_{n=1}^{N}\left\|D^{\frac{1}{2}}\left(E \mathbf{U}^{n}\right) \nabla \zeta^{n}\right\|^{2} \Delta t \\
& \leq K h_{c}^{2} \Delta t^{2}\left\|c_{\tau \tau}\right\|_{L^{2}\left(L^{2}\right)}^{2}+\left(3 \epsilon+K h_{c}^{2}\right)\|\mid \zeta\|_{L^{2}\left(L^{2}\right)}^{2} \\
&+K\left(\left\|c\left|\left\|_{L^{4}\left(H^{1}\right)}^{2}+\right\|\|\zeta\|\right|_{L^{4}\left(L^{2}\right)}^{2}\right)\|\| \mathbf{u}-\mathbf{U} \|_{L^{4}\left(L^{2}\right)}^{2}\right. \\
&+K \Delta t_{p}^{3}\left\|u_{t t}\right\|_{L^{2}\left(L^{2}\right)}^{2}\|c \mid\|_{L^{2}\left(H^{1}\right)}^{2}+h_{c}^{2}\|\| \xi\left\|_{L^{2}\left(L^{2}\right)}^{2}+h_{c}^{4}\right\|\left\|\xi_{t}\right\|_{L^{2}\left(L^{2}\right)}^{2} \\
&+K h_{c}^{2} \Delta t_{p}^{3}\left\|u_{t t}\right\|_{L^{2}\left(L^{2}\right)}^{2}\|c\|_{L^{2}\left(W_{\infty}^{1}\right)}^{2}
\end{aligned}
$$

Hence, combining (4.46) with (3.70) and (4.3) and assuming

$$
\|\mid \zeta\|_{L^{4}\left(L^{2}\right)} \leq K
$$

we have

$$
\begin{aligned}
h_{c}^{2} \| \phi^{\frac{1}{2}} \zeta^{N} & \left\|^{2}+h_{c}^{2} \sum_{n=1}^{N}\right\| D^{\frac{1}{2}}\left(E \mathbf{U}^{n}\right) \nabla \zeta^{n} \|^{2} \Delta t \\
\leq & K h_{c}^{2} \Delta t^{2}\left\|c_{\tau \tau}\right\|_{L^{2}\left(L^{2}\right)}^{2}+\left(3 \epsilon+K h_{c}^{2}\right)\|\| \|_{L^{2}\left(L^{2}\right)}^{2} \\
& +K h_{p}^{k+1}\left(\|\mathrm{u}\|_{L^{4}\left(H^{k+1}(d i v)\right)}+\|p\|_{L^{4}\left(H^{k+1}\right)}\right) \\
& +K \Delta t_{p}^{3}\left(\left\|u_{t t}\right\|_{L^{2}\left(L^{2}\right)}^{2}\|c\|_{L^{2}\left(H^{1}\right)}^{2}+h_{c}^{2}\|c\|_{L^{2}\left(W_{\infty}^{1}\right)}^{2}\right) \\
& +K h_{c}^{2(r+1)}\left[\left(h_{c}^{2}+\epsilon\right)\|c\|_{L^{2}\left(H^{r+1}\right)}^{2}+\|c\|_{H^{1}\left(H^{r-1}\right)}^{2}\right] .
\end{aligned}
$$

Combining (4.48) with the triangle inequality and using estimates for $\xi$ and lower bounds on $\phi$ and $D$ we find

$$
\begin{gathered}
h_{c}\left(\|\mid\|\left\|_{L^{\infty}\left(L^{2}\right)}+\right\|\|e\|_{L^{2}\left(H^{1}\right)}\right) \leq K\left(h_{c}^{r+1}+h_{p}^{k+1}+\Delta t_{p}^{3 / 2}+\Delta t\right) \\
+\left(\epsilon+K h_{c}^{2}\right)\|\| \|_{L^{2}\left(L^{2}\right)}
\end{gathered}
$$

Similarly, in the estimate of $h_{c}^{2}\|\| \partial_{t} \zeta \|_{L^{2}\left(L^{2}\right)}$, we have the following:

$$
h_{c}^{4} \sum_{n=1}^{N^{*}} \Delta t\left(\left|\left(\phi \frac{\check{c}^{n-1}-\hat{c}^{n-1}}{\Delta t}, \partial_{t} \zeta^{n}\right)\right|+\left|\left(\phi \frac{\check{\xi}^{n-1}-\hat{\xi}^{n-1}}{\Delta t}, \partial_{t} \zeta^{n}\right)\right|\right.
$$




$$
\begin{gathered}
\left.+\left|\left(\phi \frac{\check{\zeta}^{n-1}-\hat{\zeta}^{n-1}}{\Delta t}, \partial_{t} \zeta^{n}\right)\right|\right) \\
\leq \bar{\epsilon} h_{c}^{4}\|\| \partial_{t} \zeta\left\|_{L^{2}\left(L^{2}\right)}^{2}+K h_{c}^{2}\right\|\left\|\mathbf{u}-\mathbf{U}\left|\left\|_{L^{4}\left(L^{2}\right)}^{2}\right\|\right||c|\right\|_{L^{4}\left(H^{1}\right)}^{2} \\
+\left\|| | \zeta \left|\left\|_ { L ^ { 4 } ( L ^ { 2 } ) } ^ { 2 } \left|\|\mathbf{u}-\mathbf{U} \mid\|_{L^{4}\left(L^{2}\right)}^{2},\right.\right.\right.\right. \\
h_{c}^{4} \sum_{n=1}^{N^{*}} \Delta t\left|\left(\left[\mathbf{u}^{n}-E \mathbf{U}^{n}\right] \cdot \nabla c^{n}, \partial_{t} \zeta^{n}\right)\right| \\
\leq K h_{c}^{2} \Delta t_{p}^{3}\left\|u_{t t}\right\|_{L^{2}\left(L^{2}\right)}^{2}\left|\|c\|_{L^{2}\left(H^{1}\right)}^{2}+\bar{\epsilon} h_{c}^{4}\left\|\partial_{t} \zeta\right\|\right|_{L^{2}\left(L^{2}\right)}^{2},
\end{gathered}
$$

and

$$
\begin{aligned}
& h_{c}^{4} \sum_{n=1}^{N^{*}} \Delta t\left|\left(\left[D\left(\mathbf{u}^{n}\right)-D\left(E \mathbf{U}^{n}\right)\right] \nabla \tilde{C}^{n}, \nabla \partial_{t} \zeta^{n}\right)\right| \\
& \leq K\|\mid\| D_{\mathbf{u}}\|\|_{L^{\infty}\left(L^{\infty}\right)}\left(\Delta t_{p}^{3}\left\|u_{t t}\right\|_{L^{2}\left(L^{2}\right)}^{2}\left|\left\|c\left|\left\|_{L^{2}\left(H^{1}\right)}^{2}+\right\| \mathbf{u}-\mathbf{U}\right|\right\|_{L^{4}\left(L^{2}\right)}^{2}\right|\|c \mid\|_{L^{4}\left(H^{1}\right)}^{2}\right) \\
& \quad+\bar{\epsilon} h_{c}^{4}\left\|\partial_{t} \zeta\right\|_{L^{2}\left(L^{2}\right)}^{2} .
\end{aligned}
$$

Combining (4.50)-(4.52) and (3.77)-(3.78) we find

$h_{c}^{4} \phi_{*}|| \partial_{t} \zeta \|_{L^{2}\left(L^{2}\right)}^{2}$

$$
\begin{aligned}
\leq & K\left\{h_{c}^{2}\|\zeta\|\left\|_{L^{2}\left(L^{2}\right)}+h_{c}^{2}\right\| \xi \mid\left\|_{L^{2}\left(L^{2}\right)}^{2}+h_{c}^{2}\right\|\left\|D^{\frac{1}{2}}\left(E \mathbf{U}^{n}\right) \nabla \zeta\right\|_{L^{2}\left(L^{2}\right)}^{2}+h_{c}^{4} \Delta t^{2}\left\|c_{\tau \tau}\right\|_{L^{2}\left(L^{2}\right)}^{2}\right. \\
& \left.+h_{c}^{4}\left\|\xi_{t}\right\|_{L^{2}\left(L^{2}\right)}^{2}\right\}+4 \bar{\epsilon} h_{c}^{4}\left\|\partial_{t} \zeta \mid\right\|_{L^{2}\left(L^{2}\right)}^{2} \\
+ & K\left\{\|\mathbf{u}-\mathbf{U}\| \|_{L^{4}\left(L^{2}\right)}^{2}\left[h_{c}^{2}\|c \mid\|_{L^{4}\left(H^{1}\right)}^{2}+\|\zeta\|_{L^{4}\left(L^{2}\right)}^{2}\right]\right. \\
& +\left\|D_{\mathbf{u}}\right\| \|_{L^{\infty}\left(L^{\infty}\right)}^{2}\left(\Delta t _ { p } ^ { 3 } \| u _ { t t } \| _ { L ^ { 2 } ( L ^ { 2 } ) } ^ { 2 } \left\|c\left|\left\|_{L^{2}\left(H^{1}\right)}^{2}+\right\| \mathbf{u}-\mathbf{U}\|\|_{L^{4}\left(L^{2}\right)}^{2}\||c|\|_{L^{4}\left(H^{1}\right)}^{2}\right)\right.\right. \\
& +h_{\mathrm{c}}^{2} \Delta t_{p}^{3}\left\|u_{t t}\right\|_{L^{2}\left(L^{2}\right)}^{2}\left|\|c \mid\|_{L^{2}\left(H^{1}\right)}^{2}\right\} .
\end{aligned}
$$

Thus, substituting the approximation results for $\xi$ and $\mathbf{u}-\mathbf{U}$ and combining with (4.47) and (4.48) we have

$$
\begin{aligned}
& h_{c}^{4}\left\|\partial_{t} \zeta\right\|_{L^{2}\left(L^{2}\right)}^{2} \\
& \quad \leq K\left(h_{c}^{2(r+1)}+h_{p}^{2(k+1)}+\Delta t_{p}^{3}+\Delta t^{2}\right)+K\left(\epsilon+h_{c}^{2}\right)\|\| \|_{L^{2}\left(L^{2}\right)}^{2} .
\end{aligned}
$$

Finally, combining (4.54) and (4.49) with (4.41) and choosing $\epsilon$ and $h_{c}$ above sufficiently small, we obtain the following results.

Theorem 4.2 Assume $0<D_{*} \leq D(\mathrm{u}) \leq D^{*}$ for some constant $D_{*}, D^{*}$, and $D$ is Lipschitz continuous with respect to $\mathrm{u}$. Assume the smoothness assumptions on $\mathrm{u}$ and $\phi$ given in Lemmas 3.1 and 3.2 hold, and assume $u, p$ and $c$ satisfy the following additional smoothness assumptions, namely

$$
c \in L_{D}^{2}\left(W_{\infty}^{1}\right) \cap L_{D}^{4}\left(H^{1}\right) \cap L_{D}^{2}\left(H^{s+1}\right) \cap H^{2}\left(L^{2}\right) \cap H^{1}\left(H^{s-1}\right),
$$


for some $s \geq 1$;

$$
\mathbf{u}, p \in L_{D}^{2}\left(H^{k+1}\right) \cap L_{D}^{4}\left(H^{k+1}\right),
$$

for some $k \geq 0$ and

$$
\mathbf{u}_{t t} \in L^{2}\left(L^{2}\right) .
$$

Moreover, assume $\zeta=C-\tilde{C}$ satisfies

$$
\left\|\left|\zeta\left\|_{L^{4}\left(L^{2}\right)}^{2}+\left|\log h_{c}\right| \mid\right\| \zeta \|_{L^{2}\left(H^{1}\right)}^{2} \leq K .\right.\right.
$$

Then

$$
\|\| \|_{L^{2}\left(L^{2}\right)} \leq K\left(h_{c}^{r+1}+h_{p}^{k+1}+\Delta t_{p}^{3 / 2}+\Delta t\right),
$$

where $r=\min \left(k^{* *}, s\right)$.

Remark. A bound for $\left\|\left|\zeta\left\|_{L^{\infty}\left(L^{2}\right)}+\left|\log h_{c}\right|\right\|\right| \zeta\right\|_{L^{2}\left(H^{1}\right)}$ can be obtained by standard arguments given in [5] assuming $\left\|\xi_{t}\right\|_{L^{2}\left(L^{2}\right)}, \Delta t$, and $\Delta t_{p}^{3 / 2}$ are all $\mathcal{O}\left(\left|\log h_{c}\right|^{\frac{1}{2}}\right)$, and $\|\mid \mathbf{u}-\mathbf{U}\| \|_{L^{\infty}\left(L^{2}\right)}$ is $o\left(\left|\log h_{c}\right|\right)$. Note that $\|\mathbf{u}-\mathbf{U}\|_{L^{\infty}\left(L^{2}\right)}$ is $\mathcal{O}\left(h_{p}\right)$ if $\mathbf{u}$ and $p$ are in $L_{D}^{\infty}\left(H^{1}\right)$, and $\left\|\xi_{t}\right\|_{L^{2}\left(L^{2}\right)}$ is $\mathcal{O}\left(h_{c}\right)$ if $c_{t} \in L^{2}\left(H^{1}\right)$. 


\section{Bibliography}

[1] J. H. Bramble, A second-order finite difference analog of the first biharmonic boundary value problem, Numer. Math. 4, pp. 236-249 (1966).

[2] J. Douglas, Jr., R. E. Ewing, and M. F. Wheeler, Approximation of the pressure by a mixed method in the simulation of miscible displacement, RAIRO Anal. Numer. 17, pp. 17-33 (1983).

[3] J. Douglas, Jr. and T. F. Russell, Numerical methods for convection-dominated pdiffusion problems based on combining the method of characteristics with finite element or finite difference procedures, SIAM J. Numer. Anal. 19, pp. 871-885 (1982).

[4] T. Dupont, G. Fairweather and J. P. Johnson, Three-level Galerkin methods for parabolic equations, SIAM J. Numer. Anal. 11, pp. 392-410 (1974).

[5] R. E. Ewing, T. F. Russell, and M. F. Wheeler, Convergence analysis of an approximation of miscible displacement in porous media by mixed finite elements and a modified method of characteristics, Computer Methods in Applied Mechanics and Engineering 47, pp. 73-92 (1984).

[6] R. E. Ewing and M. F. Wheeler, Galerkin methods for miscible displacement problems with point sources and sinks-unit mobility ratio case, Mathematical Methods in Energy Research (K. I. Gross, ed.) Society for Industrial and Applied Mathematics, Philadelphia, pp. 40-58 (1984).

[7] K. W. Morton, A. Priestley, and E. Süli, Stability analysis of the LagrangeGalerkin method with non-exact integration, Report \# 86/14, Oxford University Computing Laboratory (1986).

[8] O. J. Palmer, Error estimates for finite element methods applied to contaminant transport equations, Ph. D. Thesis, Rice University (1983).

[9] O. Pironneau, On the transport-diffusion algorithm and its application to the Navier-Stokes equations, Numer. Math. 38, pp. 309-332 (1982).

[10] T. F. Russell, Time-stepping along characteristics with incomplete iteration for a Galerkin approximation of miscible displacement in porous media, SIAM J. Numer. Anal. 22, pp. 970-1013 (1985). 
[11] T. F. Russell and M. F. Wheeler, Finite element and finite difference methods for continuous flows in porous media, The Mathematics of Reservoir Simulation, R. E. Ewing, ed., Society for Industrial and Applied Mathematics, Philadelphia, pp. 35-105 (1983).

[12] E. Süli, Convergence analysis of the Lagrange-Galerkin method for the NavierStokes equations, Report \# 86/3, Oxford University Computing Laboratory (1986).

[13] M. F. Wheeler, A priori $L_{2}$ error estimates for Galerkin approximations to parabolic partial differential equations, SIAM J. Numer. Anal. 10, pp. 723-759 (1973).

[14] M. F. Wheeler and C. N. Dawson, An operator-splitting method for advectiondiffusion-reaction problems, to appear in MAFELAP Proceedings 1987. 